\title{
Localization of Fréchet Frames and Expansion of Generalized Functions
}

\author{
Stevan Pilipović ${ }^{1}$. Diana T. Stoeva ${ }^{2,3}$
}

Received: 30 September 2019 / Revised: 4 December 2020 / Accepted: 21 December 2020 /

Published online: 23 February 2021

(c) The Author(s) 2021

\begin{abstract}
Matrix-type operators with the off-diagonal decay of polynomial or sub-exponential types are revisited with weaker assumptions concerning row or column estimates, still giving the continuity results for the frame type operators. Such results are extended from Banach to Fréchet spaces. Moreover, the localization of Fréchet frames is used for the frame expansions of tempered distributions and a class of Beurling ultradistributions.
\end{abstract}

Keyword Localized frame $\cdot$ Banach frame $\cdot$ Fréchet frame $\cdot$ Tempered distributions . Ultradistributions · Frame expansions

Mathematics Subject Classification 42C15 · 46A13 • 46B15 · 46F05

\section{Introduction, Motivation and Main Aims}

Localized frames were introduced independently by Gröchenig [23] and Balan, Casazza, Heil, and Landau [2,3]. The localization conditions in [23] are related to off-diagonal decay (of polynomial or exponential type) of the matrix determined by the inner products of the frame elements and the elements of a given Riesz basis. A

Communicated by Rosihan M. Ali.

$凶 \quad$ Diana T. Stoeva

diana.stoeva@univie.ac.at; dstoeva@kfs.oeaw.ac.at

Stevan Pilipović

pilipovic@dmi.uns.ac.rs

1 Faculty of Sciences, Department of Mathematics and Informatics, University of Novi Sad, Novi Sad, Serbia

2 Faculty of Mathematics, University of Vienna, Oskar-Morgenstern-Platz 1, Vienna 1090, Austria

3 Acoustics Research Institute, Austrian Academy of Sciences, Wohllebengasse 12-14, Vienna 1040, Austria 
localized frame in this sense leads to the same type of localization of the canonical dual frame as well as to the convergence of the frame expansions in all associated Banach spaces. We refer to $[5,13,14,20,21]$, where various interesting properties and applications of localized frames were considered. The localization and self-localization, considered independently in [1-3], are directed to the over-completeness of frames and the relations between frame bounds and density with applications to Gabor frames. For the present paper, we have chosen to stick to the localization concept from [23], because the results obtained for a family of Banach spaces there can naturally be related to Fréchet frames (cf. [31-34]).

Our main aim in this paper is to present in Section 6 the frame expansions of tempered distributions and tempered ultradistributions of Beurling type by the use of localization. Matrix-type operators of Section 5 have an essential role in our investigations. The important novelty is the analysis related to sub-exponential off-diagonal decay without assumption of the exponential off-diagonal decay as it was considered in [23]. More precisely, in [23] the presumed exponential off-diagonal decay of matrices implies the analysis of sub-exponentially weighted spaces. Probably the most important impact in applications is related to the Hermite basis which is almost always used for the global expansion of $L^{2}$-functions or tempered generalized functions over $\mathbb{R}^{n}$. Our results by the use of localization, show that the same is true if one uses a kind of perturbation of Hermite functions through localization.

As particular results, not directly involved in the main ones, we extend in Sections 3 and 4 the continuity results on matrix-type operators acting on elements of a Banach or Fréchet spaces expanded by frames. We consider relaxed version of the classical off-diagonal decay conditions, assuming the column-decay and allowing row-increase in a matrix.

The paper is organized as follows. We recall in Section 2 the notation, basic definitions, and the needed known results. In Section 3, we consider matrices with column decay and possible row increase. For such type of matrices, we obtain in Section 4 continuity results for the frame related operators using less restrictive conditions in comparison with the localization conditions known in the literature. Sub-exponential localization is introduced and analyzed in Section 5. The use of Jaffard's Theorem and [23, Theorems 11 and 13] is intrinsically connected with the sub-exponential localization. Section 6 is devoted to Fréchet frames and series expansions in certain classes of Fréchet spaces based on polynomial, exponential, and sub-exponential localization. In particular, we obtain frame expansions in the Schwartz space $\mathcal{S}$ of rapidly decreasing functions and its dual, the space of tempered distributions, as well as in the spaces $\Sigma^{\alpha}, \alpha>1 / 2$, and their duals, spaces of tempered ultradistributions. In order to illustrate some results, we provide examples with the Hermite orthonormal basis $h_{n}, n \in \mathbb{N}$, and construct a Riesz basis which is polynomially and exponentially localized to $h_{n}, n \in \mathbb{N}$. Finally, in "Appendix", we add some details in the proof of the Jaffard's theorem. 


\section{Notation, Definitions, and Preliminaries}

Throughout the paper, $(\mathcal{H},\langle\cdot, \cdot\rangle)$ denotes a separable Hilbert space and $G$ (resp. $E$ ) denotes the sequence $\left(g_{n}\right)_{n=1}^{\infty}\left(\right.$ resp. $\left.\left(e_{n}\right)_{n=1}^{\infty}\right)$ with elements from $\mathcal{H}$. Recall that $G$ is called:

- frame for $\mathcal{H}[15]$ if there exist positive constants $A$ and $B$ (called frame bounds) so that $A\|f\|^{2} \leq \sum_{n=1}^{\infty}\left|\left\langle f, g_{n}\right\rangle\right|^{2} \leq B\|f\|^{2}$ for every $f \in \mathcal{H}$;

- Riesz basis for $\mathcal{H}[4]$ if its elements are the images of the elements of an orthonormal basis under a bounded bijective operator on $\mathcal{H}$.

Recall (see, e.g., [12]), if $G$ is a frame for $\mathcal{H}$, then there exists a frame $\left(f_{n}\right)_{n=1}^{\infty}$ for $\mathcal{H}$ so that

$$
f=\sum_{n=1}^{\infty}\left\langle f, f_{n}\right\rangle g_{n}=\sum_{n=1}^{\infty}\left\langle f, g_{n}\right\rangle f_{n}, f \in \mathcal{H}
$$

Such $\left(f_{n}\right)_{n=1}^{\infty}$ is called a dual frame of $\left(g_{n}\right)_{n=1}^{\infty}$. Furthermore, the analysis operator $U_{G}$, given by $U_{G} f=\left(\left\langle f, g_{n}\right\rangle\right)_{n=1}^{\infty}$, is bounded from $\mathcal{H}$ into $\ell^{2}$; the synthesis operator $T_{G}$, given by $T_{G} f=\sum_{n=1}^{\infty} c_{n} g_{n}$, is bounded from $\ell^{2}$ into $\mathcal{H}$; the frame operator $S_{G}:=T_{G} U_{G}$ is a bounded bijection of $\mathcal{H}$ onto $\mathcal{H}$ with unconditional convergence of the series $S_{G} f=\sum_{n=1}^{\infty}\left\langle f, g_{n}\right\rangle g_{n}$. The sequence $\left(S_{G}^{-1} g_{n}\right)_{n=1}^{\infty}$ is a dual frame of $\left(g_{n}\right)_{n=1}^{\infty}$, called the canonical dual of $\left(g_{n}\right)_{n=1}^{\infty}$, and it will be denoted by $\left(\tilde{g}_{n}\right)_{n=1}^{\infty}$ or $\widetilde{G}$. When $G$ is a Riesz basis of $\mathcal{H}$ (and thus a frame for $\mathcal{H}$ ), then only $\widetilde{G}$ is a dual frame of $G$, it is the unique biorthogonal sequence to $G$, and it is also a Riesz basis for $\mathcal{H}$. A frame $G$ which is not a Riesz basis has other dual frames in addition to the canonical dual and in that case we use notation $G^{d}$ or $\left(g_{n}^{d}\right)_{n=1}^{\infty}$ for a dual frame of $G$.

Next, $(X,\|\cdot\|)$ denotes a Banach space and $(\Theta,\||\cdot|\|)$ denotes a Banach sequence space; $\Theta$ is called a $B K$-space if the coordinate functionals are continuous. If the canonical vectors form a Schauder basis for $\Theta$, then $\Theta$ is called a $C B$-space. A $C B$ space is clearly a $B K$-space.

Given a $B K$-space $\Theta$ and a frame $G$ for $\mathcal{H}$ with a dual frame $G^{d}=\left(g_{n}^{d}\right)_{n=1}^{\infty}$, one associates with $\Theta$ the Banach space

$$
\mathcal{H}_{G, G^{d}}^{\Theta}:=\left\{f \in \mathcal{H}:\left(\left\langle f, g_{n}^{d}\right\rangle\right)_{n=1}^{\infty} \in \Theta,\|f\|_{\mathcal{H}_{G, G^{d}}^{\Theta}}:=\left\|\mid\left(\left\langle f, g_{n}^{d}\right\rangle\right)_{n=1}^{\infty}\right\|_{\Theta}\right\} .
$$

When $G$ is a Riesz basis for $\mathcal{H}$, then we use notation $\mathcal{H}_{G}^{\Theta}$ for $\mathcal{H}_{G, \widetilde{G}}^{\Theta}$.

\subsection{Localization of Frames}

In this paper, we consider polynomially and exponentially localized frames in the way defined in [23], and furthermore, sub-exponential localization. Let $G$ be a Riesz basis for the Hilbert space $\mathcal{H}$. A frame $E$ for $\mathcal{H}$ is called:

- polynomially localized with respect to $G$ with decay $\gamma>0$ (in short, $\gamma$-localized wrt $\left.\left(g_{n}\right)_{n=1}^{\infty}\right)$ if there is a constant $C_{\gamma}>0$ so that 


$$
\max \left\{\left|\left\langle e_{m}, g_{n}\right\rangle\right|,\left|\left\langle e_{m}, \tilde{g}_{n}\right\rangle\right|\right\} \leq C_{\gamma}(1+|m-n|)^{-\gamma}, m, n \in \mathbb{N}
$$

- exponentially localized with respect to $G$ if for some $\gamma>0$ there is a constant $C_{\gamma}>0$ so that

$$
\max \left\{\left|\left\langle e_{m}, g_{n}\right\rangle\right|,\left|\left\langle e_{m}, \widetilde{g_{n}}\right\rangle\right|\right\} \leq C_{\gamma} \mathrm{e}^{-\gamma|m-n|}, m, n \in \mathbb{N} .
$$

- $\beta$-sub-exponentially localized with respect to $G$ (for $\beta \in(0,1))$ if for some $\gamma>0$ there is $C_{\gamma}>0$ so that

$$
\max \left\{\left|\left\langle e_{m}, g_{n}\right\rangle\right|,\left|\left\langle e_{m}, \tilde{g}_{n}\right\rangle\right|\right\} \leq C_{\gamma} \mathrm{e}^{-\gamma|m-n|^{\beta}}, m, n \in \mathbb{N} .
$$

\subsection{Fréchet Frames}

We consider Fréchet spaces which are projective limits of Banach spaces as follows. Let $\left\{Y_{k},|\cdot|_{k}\right\}_{k \in \mathbb{N}_{0}}$ be a sequence of separable Banach spaces such that

$$
\begin{aligned}
& \{\boldsymbol{0}\} \neq \cap_{k \in \mathbb{N}_{0}} Y_{k} \subseteq \ldots \subseteq Y_{2} \subseteq Y_{1} \subseteq Y_{0}, \quad|\cdot|_{0} \leq|\cdot|_{1} \leq|\cdot|_{2} \leq \ldots \\
& Y_{F}:=\cap_{k \in \mathbb{N}_{0}} Y_{k} \text { is dense in } Y_{k}, \quad k \in \mathbb{N}_{0}\left(\mathbb{N}_{0}=\mathbb{N} \cup\{0\}\right) .
\end{aligned}
$$

Under the conditions (1)-(2), $Y_{F}$ is a Fréchet space and $Y_{F}^{*}$ is the inductive limit of the spaces $Y_{k}^{*}, k \in \mathbb{N}$. We will use such type of sequences in two cases: 1. $Y_{k}=X_{k}$ with norm $\|\cdot\|_{k}, k \in \mathbb{N}_{0} ; 2$. $Y_{k}=\Theta_{k}$ with norm $\|\cdot \cdot\|_{k}, k \in \mathbb{N}_{0}$.

Let $\left\{\Theta_{k},\|\mid \cdot\|_{k}\right\}_{k \in \mathbb{N}_{0}}$ be a sequence of $C B$-spaces satisfying (1). Then (2) holds, because every sequence $\left(c_{n}\right)_{n=1}^{\infty} \in \Theta_{F}$ can be written as $\sum_{n=1}^{\infty} c_{n} \delta_{n}$ with the convergence in $\Theta_{F}$, where $\delta_{n}$ denotes the $n$-th canonical vector, $n \in \mathbb{N}$. Furthermore, $\Theta_{F}^{*}$ can be identified with the sequence space $\Theta_{F}^{\circledast}:=\left\{\left(U \delta_{n}\right)_{n=1}^{\infty}: U \in \Theta_{F}^{*}\right\}$ with convergence naturally defined in correspondence with the convergence in $\Theta_{F}^{*}$.

We use the term operator for a linear mapping, and by invertible operator on $X$, we mean a bounded bijective operator on $X$. Given sequences of Banach spaces, $\left\{X_{k}\right\}_{k \in \mathbb{N}_{0}}$ and $\left\{\Theta_{k}\right\}_{k \in \mathbb{N}_{0}}$, which satisfy (1)-(2), an operator $T: \Theta_{F} \rightarrow X_{F}$ is called $F$ bounded if for every $k \in \mathbb{N}_{0}$, there exists a constant $C_{k}>0$ such that $\left\|T\left(c_{n}\right)_{n=1}^{\infty}\right\|_{k} \leq$ $C_{k}\left\|\mid\left(c_{n}\right)_{n=1}^{\infty}\right\| \|_{k}$ for all $\left(c_{n}\right)_{n=1}^{\infty} \in \Theta_{F}$.

Definition 2.1 [34] Let $\left\{X_{k},\|\cdot\|_{k}\right\}_{k \in \mathbb{N}_{0}}$ be a sequence of Banach spaces satisfying (1)-(2) and let $\left\{\Theta_{k},\||\cdot|\|_{k}\right\}_{k \in \mathbb{N}_{0}}$ be a sequence of $B K$-spaces satisfying (1)-(2). A sequence $\left(\phi_{n}\right)_{n=1}^{\infty}$ with elements from $X_{F}^{*}$ is called a General Fréchet frame (in short, General $F$-frame) for $X_{F}$ with respect to $\Theta_{F}$ if there exist sequences $\left\{\widetilde{s}_{k}\right\}_{k \in \mathbb{N}_{0}} \subseteq \mathbb{N}_{0}$, $\left\{s_{k}\right\}_{k \in \mathbb{N}_{0}} \subseteq \mathbb{N}_{0}$, which increase to $\infty$ with the property $s_{k} \leq \widetilde{s}_{k}, k \in \mathbb{N}_{0}$, and there exist constants $0<A_{k} \leq B_{k}<\infty, k \in \mathbb{N}_{0}$, satisfying

$$
\begin{aligned}
& \left(\phi_{n}(f)\right)_{n=1}^{\infty} \in \Theta_{F}, f \in X_{F}, \\
& A_{k}\|f\|_{s_{k}} \leq\|\|\left(\phi_{n}(f)\right)_{n=1}^{\infty}\left\|_{k} \leq B_{k}\right\| f \|_{\tilde{s}_{k}}, f \in X_{F}, k \in \mathbb{N}_{0},
\end{aligned}
$$

and there exists a continuous operator $V: \Theta_{F} \rightarrow X_{F}$ so that $V\left(\left(\phi_{n}(f)\right)_{n=1}^{\infty}\right)=f$ for every $f \in X_{F}$. 
When $s_{k}=\widetilde{s}_{k}=k, k \in \mathbb{N}_{0}$, and the continuity of $V$ is replaced by the stronger condition of $F$-boundedness of $V$, then the above definition reduces to the definition of a Fréchet frame (in short, $F$-frame) for $X_{F}$ with respect to $\Theta_{F}$ introduced in [32]. Although we will use in the sequel this simplified definition, Definition 2.1 is the most general one, interesting in itself, and can be considered as a non-trivial generalization of Banach frames.

In the particular case when $X_{k}=X$, and $\Theta_{k}=\Theta, k \in \mathbb{N}_{0}$, a Fréchet frame for $X_{F}$ with respect to $\Theta_{F}$ becomes a Banach frame for $X$ with respect to $\Theta$ as introduced in [22].

For another approach to frames in Fréchet spaces, we refer to [6]. For more on frames for Banach spaces, see, e.g., $[8,9,37]$ and the references therein.

\subsection{Sequence and Function Spaces}

Recall that a positive continuous function $\mu$ on $\mathbb{R}$ is called: a $k$-moderate weight if $k \geq 0$ and there exists a constant $C>0$ so that $\mu(t+x) \leq C(1+|t|)^{k} \mu(x), t, x \in \mathbb{R}$; a $\beta$-sub-exponential (resp. exponential) weight, if $\beta \in(0,1)$ (resp. $\beta=1$ ) and there exist constants $C>0, \gamma>0$, so that $\mu(t+x) \leq C e^{\gamma|t|^{\beta}} \mu(x), t, x \in \mathbb{R}$. If $\beta$ is clear from the context, we will write just sub-exponential weight. Let $\mu$ be a $k$ moderate, sub-exponential, or exponential weight so that $\mu(n) \geq 1$ for every $n \in \mathbb{N}$, and $p \in[1, \infty)$. Then the Banach space

$$
\ell_{\mu}^{p}:=\left\{\left(a_{n}\right)_{n=1}^{\infty}:\left\|\mid\left(a_{n}\right)_{n}\right\| \|_{p, \mu}:=\left(\sum_{n=1}^{\infty}\left|a_{n}\right|^{p} \mu(n)^{p}\right)^{1 / p}<\infty\right\}
$$

is a $C B$-space. We refer, for example, to [28, Ch. 27] for the so-called Köthe sequence spaces.

We will need the following lemma, which can be easily proved by the use of [32, Theor. 4.2].

Lemma 2.2 Let $G$ be a frame for $\mathcal{H}$ and let $G^{d}=\left(g_{n}^{d}\right)_{n=1}^{\infty}$ be a dual frame of $G$. Let $\mu_{k}$ be $k$-moderate (resp. sub-exponential or exponential) weights, $k \in \mathbb{N}_{0}$, so that

$$
1=\mu_{0}(x) \leq \mu_{1}(x) \leq \mu_{2}(x) \leq \ldots, \text { for every } x \in \mathbb{R} .
$$

Then the spaces $\Theta_{k}:=\ell_{\mu_{k}}^{2}, k \in \mathbb{N}_{0}$, satisfy (1)-(2). Denote $M:=\left\{\left(\left\langle f, g_{n}^{d}\right\rangle\right)_{n=1}^{\infty}\right.$ : $f \in \mathcal{H}\}$. The assumption that $M \cap \Theta_{F}$ is dense in $M \cap \Theta_{k} \neq\{0\}$ with respect to the $\|\mid \cdot\|_{k}$-norm for every $k \in \mathbb{N}$, leads to the conclusion that the spaces $X_{k}:=\mathcal{H}_{G, G^{d}}^{\Theta_{k}}$, $k \in \mathbb{N}$, satisfy (1)-(2).

If $G$ is a Riesz basis for $\mathcal{H}$, then the density assumption of $M \cap \Theta_{F}$ in $M \cap \Theta_{k} \neq\{0\}$, $k \in \mathbb{N}$, is fulfilled and in addition one has that $g_{n} \in X_{F}$ for every $n \in \mathbb{N}$.

Throughout the paper, we also consider specific weights, relevant to the function spaces of interest and the corresponding sequence spaces. Let $k \in \mathbb{N}_{0}$ and $\mu_{k}(x)=$ $(1+|x|)^{k}$ (resp. $\left.\mu_{k}(x)=e^{k|x|^{\beta}}, \beta \in(0,1]\right), x \in \mathbb{R}$. Then, with $\Theta_{k}:=\ell_{\mu_{k}}^{p}, k \in \mathbb{N}_{0}$, 
the projective limit $\cap_{k \in \mathbb{N}_{0}} \Theta_{k}$ is the space $\mathbf{s}$ of rapidly (resp. $\mathfrak{s}^{\beta}$ of sub-exponentially when $\beta<1$ and exponentially when $\beta=1$ ) decreasing sequences determined by $\left.\left\{\left(a_{n}\right)_{n=1}^{\infty} \in \mathbb{C}^{\mathbb{N}}:\left.\left(\sum_{n=1}^{\infty} \mid a_{n} \mu_{k}(n)\right)\right|^{p}\right)^{1 / p}<\infty, \forall k \in \mathbb{N}_{0}\right\}$, which is the same set for any $p \in[1, \infty)$. The space $\mathbf{s}$ (resp. $\mathfrak{s}^{\beta}$ ) can also be derived as the projective limit of the Banach spaces $\mathbf{s}_{k}$ (resp. $\mathfrak{s}_{k}^{\beta}$ ) defined as $\left\{\left(a_{n}\right)_{n=1}^{\infty} \in \mathbb{C}^{\mathbb{N}}:\left\|\mid\left(a_{n}\right)_{n=1}^{\infty}\right\| \|_{\text {sup }, k}:=\right.$ $\left.\sup _{n \in \mathbb{N}}\left|a_{n}\right| n^{k}<\infty\right\}$, (resp. $\left.\left.\|\left.|\cdot|\right|_{\text {sup }, k} ^{\beta}:=\sup _{n \in \mathbb{N}}\left|a_{n}\right| e^{k n^{\beta}}<\infty\right\}\right), k \in \mathbb{N}_{0}$; note that here instead of $k \in\{0,1,2,3, \ldots\}$ one can also use any strictly increasing sequence of nonnegative numbers $k \in\left\{0, q_{1}, q_{2}, q_{3}, \ldots\right\}$.

Recall that the well-known Schwartz space $\mathcal{S}$ is the intersection of Banach spaces

$$
\mathcal{S}_{k}(\mathbb{R}):=\left\{f \in L^{2}(\mathbb{R}):\|f\|_{k}=\sum_{m=0}^{k}\left\|\left(1+|\cdot|^{2}\right)^{k / 2} f^{(m)}\right\|_{L^{2}(\mathbb{R})}\right\}, k \in \mathbb{N} .
$$

The dual $\mathcal{S}^{\prime}(\mathbb{R})$ is the space of tempered distributions.

The space of sub-exponentially decreasing functions of order $1 / \alpha, \alpha>1 / 2$, is $\Sigma^{\alpha}:=$ $X_{F}=\cap_{k \in \mathbb{N}_{0}} \Sigma^{k, \alpha}$ where $\Sigma^{k, \alpha}$ are Banach spaces of $L^{2}-$ functions with finite norms

$$
\|f\|_{k}^{\alpha}=\sup _{n \in \mathbb{N}_{0}}\left\|\frac{k^{n} e^{k|x|^{1 / \alpha}}\left|f^{(n)}(x)\right|}{n !^{\alpha}}\right\|_{L^{2}(\mathbb{R})}<\infty, k \in \mathbb{N} .
$$

Its dual $\left(\Sigma^{\alpha}(\mathbb{R})\right)^{\prime}$ is the space of Beurling tempered ultradistributions, cf. [19,30].

Remark 2.3 The case $\alpha=1 / 2$ leads to the trivial space $\Sigma^{1 / 2}=\{0\}$. There is another way in considering the test space which corresponds to that limiting Beurling case $\alpha=1 / 2$ and can be considered also for $\alpha<1 / 2$ (cf. $[10,11,19,29]$ ). We will not treat these cases in the current paper.

In the sequel, $\left(h_{n}\right)_{n=1}^{\infty}$ is the Hermite orthonormal basis of $L^{2}(\mathbb{R})$ re-indexed from 1 to $\infty$ instead of from 0 to $\infty$. Recall that $h_{n} \in \Sigma^{\alpha}, \alpha>1 / 2, n \in \mathbb{N}$. Moreover, we know [36]:

- If $f \in \mathcal{S}$, then $\left(\left\langle f, h_{n}\right\rangle\right)_{n=1}^{\infty} \in \mathbf{s}$; conversely, if $\left(a_{n}\right)_{n=1}^{\infty} \in \mathbf{s}$, then $\sum_{n=1}^{\infty} a_{n} h_{n}$ converges in $\mathcal{S}$ to some $f$ with $\left(\left\langle f, h_{n}\right\rangle\right)_{n=1}^{\infty}=\left(a_{n}\right)_{n=1}^{\infty}$.

- If $F \in \mathcal{S}^{\prime}$, then $\left(b_{n}\right)_{n=1}^{\infty}:=\left(F\left(h_{n}\right)\right)_{n=1}^{\infty} \in \mathbf{s}^{\prime}$ and $F(f)=\sum_{n=1}^{\infty}\left\langle f, h_{n}\right\rangle b_{n}, f \in \mathcal{S}$; conversely, if $\left(b_{n}\right)_{n=1}^{\infty} \in \mathbf{s}^{\prime}$, then the mapping $F: f \rightarrow \sum_{n=1}^{\infty}\left\langle f, h_{n}\right\rangle b_{n}$ is well defined on $\mathcal{S}$, it determines $F$ as an element of $\mathcal{S}^{\prime}$ and $\left(F\left(h_{n}\right)\right)_{n=1}^{\infty}=\left(b_{n}\right)_{n=1}^{\infty}$.

The above two statements also hold when $\mathcal{S}, \mathcal{S}^{\prime}, \mathbf{s}$, and $\mathbf{s}^{\prime}$ are replaced by $\Sigma^{\alpha},\left(\Sigma^{\alpha}\right)^{\prime}$, $\mathfrak{s}^{1 /(2 \alpha)}$, and $\left(\mathfrak{s}^{1 /(2 \alpha)}\right)^{\prime}$ with $\alpha>1 / 2$, respectively $[19,29,30]$.

We can consider $\mathcal{S}$ and $\Sigma^{\alpha}$ as the projective limit of Hilbert spaces $\mathcal{H}^{k}, k \in \mathbb{N}_{0}$, with elements $f=\sum_{n} a_{n} h_{n}$, in the first case with norms

$$
\left.\|f\|_{\mathcal{H}^{k}}:=\left\|\mid\left(a_{n} n^{k}\right)_{n}\right\|_{\ell^{2}}<\infty\right\}, k \in \mathbb{N}_{0},
$$

and in the second case with norms

$$
\left.\|f\|_{\mathcal{H}^{k}}:=\left\|\mid\left(a_{n} e^{k n^{1 /(2 \alpha)}}\right)_{n}\right\|_{\ell^{2}}<\infty\right\}, k \in \mathbb{N}_{0} .
$$


Thus, $\left(h_{n}\right)_{n}$ is an $F$-frame for $\mathcal{S}(\mathbb{R})$ with respect to $\mathbf{s}$ as well as an $F$-frame for $\Sigma^{\alpha}$ with respect to $\mathfrak{s}^{1 / 2 \alpha}, \alpha>1 / 2,(F$ - boundedness is trivial $)$.

\section{Matrix-Type Operators}

Papers $[14,21,23]$ concern matrices with off-diagonal decay of the form: for some $\gamma>0$ there is $C_{\gamma}>0$ such that

$$
\left|A_{m, n}\right| \leq \frac{C_{\gamma}}{(1+|m-n|)^{\gamma}} \quad\left(\text { resp. }\left|A_{m, n}\right| \leq C_{\gamma} \mathrm{e}^{-\gamma|m-n|}\right), \forall n, m \in \mathbb{N}
$$

As we noted in the introduction, matrix operators in this section and the next one are not essentially related to Sections 5 and 6 . But they significantly illuminate such operators in our main results. Moreover, we refer in Section 4 to results of Section 3 and in Remark 6.4 we refer to Section 4.

We will consider matrices with more general off-diagonal type of decay (see $(* * *)$ below which is weaker condition compare to the polynomial type condition in (6)). Moreover, we consider matrices which have column decrease but allow row increase (see Propositions 3.2 and 3.6) allowing sub-exponential type conditions as well. For such more general matrices, we generalize some results from [23] with respect to certain Banach spaces and, furthermore, proceed to the Fréchet case.

In the sequel, for a given matrix $\left(A_{m n}\right)_{m, n \in \mathbb{N}}$, the letter $\mathcal{A}$ will denote the mapping $\left(c_{n}\right)_{n=1}^{\infty} \rightarrow\left(a_{m}\right)_{m=1}^{\infty}$ determined by $a_{m}=\sum_{n=1}^{\infty} A_{m, n} c_{n}$ (assuming convergence), $m \in \mathbb{N}$; conversely, for a given mapping $\mathcal{A}$ determined on a sequence space containing the canonical vectors $\delta_{n}, n \in \mathbb{N}$, the corresponding matrix $\left(A_{m n}\right)_{m, n \in \mathbb{N}}$ is given by $A_{m, n}=\left\langle\mathcal{A} \delta_{n}, \delta_{m}\right\rangle$. We will sometimes use $\mathcal{A}$ with the meaning of $\left(A_{m n}\right)_{m, n \in \mathbb{N}}$ and vice-verse.

\subsection{Polynomial-Type Conditions}

Let us begin with some comparison of polynomial type of off-diagonal decay:

Lemma 3.1 Let $\gamma>0$. Consider the following conditions:

$$
\begin{aligned}
& (*)\left|A_{m, n}\right| \leq C\left\{\begin{array}{l}
\frac{(1+m)^{\gamma}}{(1+n)^{2 \gamma}}, n \geq m, \\
\frac{(1+n)^{\gamma}}{(1+m)^{2 \gamma}}, n \leq m, \text { for some } C>0 .
\end{array}\right. \\
& (* *)\left|A_{m, n}\right| \leq C(1+|n-m|)^{-\gamma}, \text { for some } C>0 .
\end{aligned}
$$

Then, the implications $(*) \Rightarrow(* *) \Rightarrow(* * *)$ hold. The converse implications are not valid. 
Proof Implications $(*) \Rightarrow(* *) \Rightarrow(* * *)$ follow from the inequalities $\frac{(1+\min (m, n))^{\gamma}}{(1+\max (m, n))^{2 \gamma}}$ $\leq(1+|n-m|)^{-\gamma} \leq \frac{(\min (m, n))^{\gamma}}{(\max (m, n))^{\gamma}}, n, m \in \mathbb{N}$, which are easy to be verified. To show that $(* * *)$ does not imply $(* *)$ even up to a multiplication with a constant, take a matrix $A_{m, n}$ which satisfies $\left|A_{m, n}\right|=\frac{C n^{\gamma}}{m^{\gamma}}, n \leq m$, for some $\gamma>0$ and some positive constant $C$, and assume that there exist $\gamma_{1}(\gamma) \in \mathbb{N}$ and a positive constant $K$ so that for $m \geq n$ one has $\frac{C n^{\gamma}}{m^{\gamma}} \leq \frac{K}{(1+m-n)^{\gamma_{1}}}$; then taking $m=2 n$, one obtains $0<C \cdot 2^{-\gamma} \leq \frac{K}{(1+n)^{\gamma_{1}}} \rightarrow 0$ as $n \rightarrow \infty$, which leads to a contradiction. In a similar spirit, one can show that $(* *)$ does not imply $(*)$.

Below we show that the relaxed polynomial-type conditions, as well as conditions allowing row-increase, still lead to continuous operators.

Proposition 3.2 Assume that the matrix $\left(A_{m n}\right)_{m, n \in \mathbb{N}}$ satisfies the condition

$$
\left|A_{m, n}\right| \leq \begin{cases}C_{0} n^{\gamma_{0}}, & n>m \\ C_{1} n^{\gamma_{1}} m^{-\gamma_{1}}, & n \leq m\end{cases}
$$

for some $\gamma_{0} \geq 0, \gamma_{1}>0, C_{0}>0, C_{1}>0$. Then $\mathcal{A}$ is a continuous operator from $\mathbf{s}_{\gamma_{1}+\gamma_{0}+1+\varepsilon}$ into $\mathbf{s}_{\gamma_{1}}$ for any $\varepsilon \in(0,1]$.

Proof Let $\varepsilon \in(0,1]$ and let $\left(c_{n}\right)_{n=1}^{\infty} \in \mathbf{s}_{\gamma_{0}+\gamma_{1}+1+\varepsilon}$. For every $n>m$,

$$
\left|A_{m, n} c_{n}\right| \leq C_{0}\left|c_{n}\right| n^{\gamma_{0}} \leq C_{0}\left(\sup _{j}\left(\left|c_{j}\right| j^{\gamma_{0}+\gamma_{1}+1+\varepsilon}\right)\right) \frac{1}{n^{\gamma_{1}+1+\varepsilon}}
$$

Next,

$$
\begin{aligned}
\left|\sum_{n=1}^{m} A_{m, n} c_{n}\right| & \leq C_{1} m^{-\gamma_{1}} \sum_{n=1}^{m}\left|c_{n}\right| n^{\gamma_{1}} \\
& \leq C_{1} m^{-\gamma_{1}}\left\|\left|\left(c_{n}\right)_{n=1}^{\infty} \|\right|_{\text {sup }, \gamma_{0}+\gamma_{1}+1+\varepsilon} \sum_{n=1}^{m} \frac{1}{n \gamma_{0}+1+\varepsilon} .\right.
\end{aligned}
$$

Therefore,

$$
\begin{aligned}
\left|a_{m}\right| \leq & \left|\sum_{n=1}^{m} A_{m, n} c_{n}\right|+\left|\sum_{n=m+1}^{\infty} A_{m, n} c_{n}\right| \\
\leq & C_{1} m^{-\gamma_{1}}\left\|\mid\left(c_{n}\right)_{n=1}^{\infty}\right\| \|_{\text {sup }, \gamma_{0}+\gamma_{1}+1+\varepsilon} \sum_{n=1}^{\infty} \frac{1}{n^{\gamma_{0}+1+\varepsilon}} \\
& +C_{0}\left\|\left|\left(c_{n}\right)_{n=1}^{\infty} \|\right|_{\text {sup }, \gamma_{0}+\gamma_{1}+1+\varepsilon} \sum_{n=m+1}^{\infty} \frac{1}{n^{\gamma_{1}+1+\varepsilon}} .\right.
\end{aligned}
$$

Since $\sum_{n=m+1}^{\infty} \frac{1}{n^{\gamma_{1}+1+\varepsilon}} \leq m^{-\gamma_{1}} \sum_{n=m+1}^{\infty} \frac{1}{n^{1+\varepsilon}}$, the assertion follows. 
A direct consequence of Proposition 3.2 is:

Corollary 3.3 Assume that the matrix $\left(A_{m n}\right)_{m, n \in \mathbb{N}}$ satisfies: there exist $\gamma_{0} \geq 0$ and $C_{0}>0$, and for every $\gamma>0$ there is $C_{\gamma}>0$ so that

$$
\left|A_{m, n}\right| \leq \begin{cases}C_{0} n^{\gamma_{0}}, & n>m \\ C_{\gamma} n^{\gamma} m^{-\gamma}, & n \leq m .\end{cases}
$$

Then $\mathcal{A}$ is a continuous operator from $\mathbf{s}$ into $\mathbf{s}$.

In order to determine $\mathcal{A}$ as a mapping from a space $\mathbf{s}_{\gamma_{1}}$ into the same space, we have to change the decay condition.

Proposition 3.4 Let $\left(A_{m n}\right)_{m, n \in \mathbb{N}}$ satisfy:

$$
\begin{gathered}
\left(\exists \varepsilon>0, \gamma_{1} \in \mathbb{N}\right)\left(\exists C_{0}>0, C_{1}>0\right) \text { such that } \\
\quad\left|A_{m, n}\right| \leq \begin{cases}C_{0} n^{-1-\varepsilon}, & n>m, \\
C_{1} n^{\gamma_{1}} m^{-\gamma_{1}-1-\varepsilon}, & n \leq m .\end{cases}
\end{gathered}
$$

Then $\mathcal{A}$ is a continuous operator from $\mathbf{s}_{\gamma_{1}}$ into $\mathbf{s}_{\gamma_{1}}$.

Remark 3.5 For the same conclusion as above, one has in [23] another condition noncomparable to (7):

$$
\left|A_{m, n}\right| \leq C(1+|n-m|)^{-\gamma_{1}-1-\varepsilon} .
$$

\subsection{Sub-exponential- and Exponential-Type Conditions}

Up to the end of the paper $\beta$ will be a fixed number of the interval $(0,1] ; \beta=1$ is related to the exponential growth order while $\beta \in(0,1)$ corresponds to the pure sub-exponential growth order.

Proposition 3.6 Assume that the matrix $\left(A_{m n}\right)_{m, n \in \mathbb{N}}$ satisfies the condition: There exist positive constants $C_{0}, C_{1}$ and $\gamma_{0} \geq 0, \gamma_{1}>0$, so that

$$
\left|A_{m, n}\right| \leq \begin{cases}C_{0} \mathrm{e}^{\gamma_{0} n^{\beta}}, & n>m, \\ C_{1} \mathrm{e}^{-\gamma_{1}\left(m^{\beta}-n^{\beta}\right)}, & n \leq m .\end{cases}
$$

Then $\mathcal{A}$ is a continuous operator from $\mathfrak{s}_{\gamma_{1}+\gamma_{0}+\varepsilon}^{\beta}$ into $\mathfrak{s}_{\gamma_{1}}^{\beta}$ for any $\varepsilon \in(0,1)$.

Proof Let $\varepsilon \in(0,1)$ and let $\left(c_{n}\right)_{n=1}^{\infty} \in \mathfrak{s}_{\gamma_{1}+\gamma_{0}+\varepsilon}^{\beta}$. Then for $n>m$,

$$
\left|A_{m, n} c_{n}\right| \leq C_{0}\left|c_{n}\right| \mathrm{e}^{\gamma_{0} n^{\beta}} \leq C_{0}\left(\sup _{j}\left|c_{j}\right| \mathrm{e}^{\left(\gamma_{1}+\gamma_{0}+\varepsilon\right) j^{\beta}}\right) \mathrm{e}^{-\left(\gamma_{1}+\varepsilon\right) n^{\beta}}
$$


Further on,

$$
\begin{aligned}
\left|\sum_{n=1}^{m} A_{m, n} c_{n}\right| & \leq C_{1} \sum_{n=1}^{m}\left|c_{n}\right| \mathrm{e}^{\gamma_{1}\left(n^{\beta}-m^{\beta}\right)} \\
& \leq C_{1} \mathrm{e}^{-\gamma_{1} m^{\beta}}\left(\sup _{j \in \mathbb{N}}\left|c_{j}\right| \mathrm{e}^{\left(\gamma_{1}+\gamma_{0}+\varepsilon\right) j^{\beta}}\right) \sum_{n=1}^{m} \mathrm{e}^{-\left(\gamma_{0}+\varepsilon\right) n^{\beta}} .
\end{aligned}
$$

Therefore,

$$
\left|a_{m}\right| \leq \mathrm{e}^{-\gamma_{1} m^{\beta}}\left(C_{1} \sum_{n=1}^{\infty} \mathrm{e}^{-\left(\gamma_{0}+\varepsilon\right) n^{\beta}}+C_{0} \sum_{n=1}^{\infty} \mathrm{e}^{-\varepsilon n^{\beta}}\right)\left\|\left|\left(c_{n}\right)_{n} \|\right|_{\text {sup }, \gamma_{1}+\gamma_{0}+\varepsilon}^{\beta} .\right.
$$

This completes the proof.

Remark 3.7 Since $e^{-\gamma(m-n)^{\beta}} \leq e^{-\gamma\left(m^{\beta}-n^{\beta}\right)}$ for $n \leq m(\beta \in(0,1], \gamma \in(0, \infty))$, in (9) we consider $e^{-\gamma\left(m^{\beta}-n^{\beta}\right)}$ instead of $e^{-\gamma(m-n)^{\beta}}$.

As a consequence of Proposition 3.6, we have:

Corollary 3.8 Assume that the matrix $\left(A_{m n}\right)_{m, n \in \mathbb{N}}$ satisfies the condition: There exist constants $C_{0}>0$ and $\gamma_{0} \geq 0$, and for every $\gamma>0$, there is a positive constant $C_{\gamma}$ so that

$$
\left|A_{m, n}\right| \leq \begin{cases}C_{0} e^{\gamma_{0} n^{\beta}}, & n>m \\ C_{\gamma} e^{\gamma\left(n^{\beta}-m^{\beta}\right)}, & n \leq m .\end{cases}
$$

Then $\mathcal{A}$ is a continuous operator from $\mathfrak{s}^{\beta}$ into $\mathfrak{s}^{\beta}$.

Proposition 3.9 Let $\left(A_{m n}\right)_{m, n \in \mathbb{N}}$ satisfy the condition: There exist positive constants $\varepsilon, \gamma_{1}, C_{0}, C_{1}$, so that

$$
\left|A_{m, n}\right| \leq \begin{cases}C_{0} e^{-\varepsilon n^{\beta}}, & n>m \\ C_{1} e^{\gamma_{1} n^{\beta}} e^{-\left(\gamma_{1}+\varepsilon\right) m^{\beta}}, & n \leq m\end{cases}
$$

Then $\mathcal{A}$ is a continuous operator from $\mathfrak{s}_{\gamma_{1}}^{\beta}$ into $\mathfrak{s}_{\gamma_{1}}^{\beta}$.

Remark 3.10 One can simply show that the assumption $\left|A_{m, n}\right| \leq C e^{-\gamma|m-n|^{\beta}}, m, n \in$ $\mathbb{N}$, leads to similar continuity results. We will consider this condition later in relation to the invertibility of such matrices and the Jaffard theorem.

\section{Continuity of the Frame-Related Operators Under Relaxed "Decay" Conditions}

We now determine weaker localization conditions which are still sufficient to imply continuity of the frame-related operators. 
Proposition 4.1 Let $G$ be a frame for $\mathcal{H}, G^{d}$ be a dual frame of $G$, and $\mu_{k}(x)=$ $(1+|x|)^{k}, k \in \mathbb{N}_{0}$. Under the notations in Lemma 2.2, assume that $M \cap \Theta_{F}$ is dense in $M \cap \Theta_{k} \neq\{0\}$ with respect to the $\|\mid \cdot\|_{k}$-norm for every $k \in \mathbb{N}$ and let $E=\left(e_{n}\right)_{n=1}^{\infty}$ be a sequence with elements from $X_{F}$ which is a frame for $\mathcal{H}$. Then the following statements hold.

(i) Assume that there exist $s_{0} \in \mathbb{N}, C>0$ and for every $k \in \mathbb{N}$ there exists $C_{k}>0$ such that

$$
\left|\left\langle e_{m}, g_{n}\right\rangle\right| \leq \begin{cases}C n^{s_{0}}, & n>m \\ C_{k} n^{k} m^{-k}, & n \leq m\end{cases}
$$

Then the analysis operator $f \rightarrow U_{E} f=\left(\left\langle f, e_{m}\right\rangle\right)_{m=1}^{\infty}$ is continuous from $X_{F}$ into $\mathbf{s}$.

(ii) Assume that there exist $\widetilde{s}_{0} \in \mathbb{N}_{0}, \widetilde{C}>0$ and for every $k \in \mathbb{N}$ there exists $\widetilde{C}_{k}>0$ such that

$$
\left|\left\langle e_{m}, g_{n}^{d}\right\rangle\right| \leq \begin{cases}\widetilde{C} m^{\widetilde{s_{0}}}, & m>n \\ \widetilde{C}_{k} m^{k} n^{-k}, & m \leq n\end{cases}
$$

Then the synthesis operator $\left(c_{n}\right)_{n} \rightarrow T_{E}\left(c_{n}\right)=\sum c_{n} e_{n}$ is continuous from $\mathbf{s}$ into $X_{F}$.

(iii) Under the assumptions of (i) and (ii), the frame operator $T_{E} U_{E}$ is continuous from $X_{F}$ into $X_{F}$.

Proof Note that under the given assumptions, $\Theta_{F}$ is the space $\mathbf{s}$.

(i) Let $A_{m, n}=\left\langle g_{n}, e_{m}\right\rangle, m, n \in \mathbb{N}$, and $\mathcal{A}$ be the corresponding operator for the matrix $A$. Let $f \in X_{F}$. Then $\left(\left\langle f, g_{n}^{d}\right\rangle\right)_{n=1}^{\infty} \in \mathbf{s}$ and

$$
\mathcal{A}\left(\left\langle f, g_{n}^{d}\right\rangle\right)_{n=1}^{\infty}=\left(\sum_{n=1}^{\infty}\left\langle g_{n}, e_{m}\right\rangle\left\langle f, g_{n}^{d}\right\rangle\right)_{m=1}^{\infty}=\left(\left\langle f, e_{m}\right\rangle\right)_{m=1}^{\infty}
$$

By Corollary 3.3, it follows that $\left(\left\langle f, e_{m}\right\rangle\right)_{n=1}^{\infty} \in \mathbf{s}$. Furthermore, by Proposition 3.2 , for every $k \in \mathbb{N}$, there is a constant $K_{s_{0}, k, C, C_{k}}$ so that

$$
\begin{aligned}
\|\|\left(\left\langle f, e_{m}\right\rangle\right)_{m} \|_{\text {sup }, k} & =\left\|\left|\mathcal{A}\left(\left\langle f, g_{n}^{d}\right\rangle\right)_{n}\|\|_{\text {sup }, k} \leq K_{S_{0}, k, C, C_{k}}\left\|\mid\left(\left\langle f, g_{n}^{d}\right\rangle\right)_{n}\right\| \|_{\text {sup }, s_{0}+k+2}\right.\right. \\
& \leq K_{s_{0}, k, C, C_{k}}\|\|\left(\left\langle f, g_{n}^{d}\right\rangle\right)_{n}\left\|_{\Theta_{s_{0}+k+2}}=K_{s_{0}, k, C, C_{k}}\right\| f \|_{s_{0}+k+2}
\end{aligned}
$$

Therefore, the analysis operator $U_{E}$ is continuous from $X_{F}$ into $\mathbf{s}$.

(ii) Let $\left(c_{n}\right) \in \mathbf{s}$. First we show that $\sum_{n=1}^{\infty} c_{n} e_{n}$ converges in $X_{F}$ and then the continuity of $T_{E}$. Since $\left(c_{n}\right)_{n=1}^{\infty} \in \ell^{2}$, we have $x=\sum_{n} c_{n} e_{n} \in \mathcal{H}$. Denote $A_{m, n}=\left\langle e_{n}, g_{m}^{d}\right\rangle$ and consider the corresponding operator $\mathcal{A}$. Then $\left(\left\langle x, g_{m}^{d}\right\rangle\right)_{m}=$ $\left(\sum_{n} A_{m, n} c_{n}\right)_{m}=\mathcal{A}\left(c_{n}\right) \in \mathbf{s}$ (by Corollary 3.3), which implies that $x \in X_{F}$, and furthermore, for every $k \in \mathbb{N}$, one has $\left\|T_{E}\left(c_{n}\right)_{n}\right\|_{k}=\|x\|_{k}=\left\|\mid\left(\left\langle x, g_{m}^{d}\right\rangle\right)_{m}\right\| \|_{\Theta_{k}}$. 
For every $k \in \mathbb{N}$, there is a constant $R_{k}$ such that $\left\|\left|\left(d_{n}\right)\|\|_{\Theta_{k}} \leq R_{k}\left\|\mid\left(d_{n}\right)\right\| \|_{\text {sup }, k+2}\right.\right.$ for every $\left(d_{n}\right) \in \mathbf{s}_{k+2}$. By Proposition 3.2, we conclude that

$$
\begin{aligned}
\left\|T_{E}\left(c_{n}\right)_{n}\right\|_{k} & \leq R_{k}\left\|\left(\left\langle x, g_{m}^{d}\right\rangle\right)_{m}\right\|\left\|_{\text {sup }, k+2}=R_{k}\right\| \mathcal{A}\left(c_{n}\right)\|\|_{\text {sup }, k+2} \\
& \leq R_{k} K_{\left(s_{0}, k, C, C_{k}\right)}\left\|\left(c_{n}\right)\right\|_{\text {sup }, s_{0}+k+4} .
\end{aligned}
$$

Thus, the synthesis operator $T_{E}$ is well defined and continuous from $\mathbf{s}$ into $X_{F}$. (iii) follows from (i) and (ii).

It is of interest to consider the case when $X_{F}$ is $\mathcal{S}$.

Corollary 4.2 Let $\left(e_{n}\right)_{n=1}^{\infty}$ be a frame of $L^{2}(\mathbb{R})$ with elements in $\mathcal{S}(\mathbb{R})$. Assume that for every $k \in \mathbb{N}$ there are constants $C_{k}, \widetilde{C}_{k}$ such that

$$
\left|\left\langle e_{m}, h_{n}\right\rangle\right| \leq\left\{\begin{array}{l}
C_{k} m^{k} n^{-k}, n>m, \\
\widetilde{C}_{k} n^{k} m^{-k}, n \leq m .
\end{array}\right.
$$

Then the analysis operator $U_{E}$ is continuous from $\mathcal{S}$ into $\mathbf{s}$, the synthesis operator $T_{E}$ is continuous from $\mathbf{s}$ into $\mathcal{S}$, and the frame operator $T_{E} U_{E}$ is continuous from $\mathcal{S}$ into $\mathcal{S}$.

Now, we consider sub-exponential weights.

Proposition 4.3 Let $\beta \in(0,1)$ and let the assumptions of the first part of Lemma 2.2 hold with the weights $\mu_{k}(x)=e^{k|x|^{\beta}}, k \in \mathbb{N}_{0}$. Let $E=\left(e_{n}\right)_{n=1}^{\infty}$ be a sequence with elements from $X_{F}$ which is a frame for $\mathcal{H}$. Then the following statements hold.

(i) Assume that there exist constants $\gamma_{0} \in \mathbb{N}, C>0$ such that for every $k \in \mathbb{N}$ there exists $C_{k}>0$ such that

$$
\left|\left\langle e_{m}, g_{n}\right\rangle\right| \leq \begin{cases}C \mathrm{e}^{\gamma_{0} n^{\beta}}, & n>m, \\ C_{k} \mathrm{e}^{k\left(n^{\beta}-m^{\beta}\right)}, & n \leq m, k \in \mathbb{N}\end{cases}
$$

Then the analysis operator $f \rightarrow U_{E} f=\left(\left\langle f, e_{m}\right\rangle\right)_{m=1}^{\infty}$ is continuous from $X_{F}$ into $\mathfrak{s}^{\beta}$.

(ii) Assume that there exist constants $\tilde{\gamma}_{0} \in \mathbb{N}, \tilde{C}>0$ such that for every $k \in \mathbb{N}$ there exists $\tilde{C}_{k}>0$ such that

$$
\left|\left\langle e_{m}, g_{n}^{d}\right\rangle\right| \leq \begin{cases}\tilde{C} \mathrm{e}^{\tilde{\gamma}_{0} m^{\beta}}, & m>n \\ \tilde{C}_{k} \mathrm{e}^{k\left(m^{\beta}-n^{\beta}\right)}, & m \leq n\end{cases}
$$

Then the synthesis operator $\left(c_{n}\right)_{n} \rightarrow T_{E}\left(c_{n}\right)=\sum c_{n} e_{n}$ is continuous from $\mathfrak{s}^{\beta}$ into $X_{F}$.

(iii) If (11) and (12) hold, then the frame operator $T_{E} U_{E}$ is continuous from $X_{F}$ into $X_{F}$. 
Proof Under the given assumptions, $\Theta_{F}$ is the space $\mathfrak{s}^{\beta}$. The rest of the proof can be done in a similar way as the proof of Proposition 4.1, using Corollary 3.8 instead of Corollary 3.3.

If in the above proposition one chooses $G$ to be the Hermite basis $\left(h_{n}\right)_{n=1}^{\infty}$ and $\beta=1 /(2 \alpha), \alpha>1 / 2$, then $X_{F}=\Sigma^{\alpha}$.

Corollary 4.4 Let $\alpha>1 / 2$. Let $\left(e_{n}\right)_{n=1}^{\infty}$ be a sequence with elements from $\Sigma^{\alpha}$ which is a frame for $L^{2}(\mathbb{R})$ and such that for every $k \in \mathbb{N}$ there are constants $C_{k}, \widetilde{C}_{k}$ such that

$$
\left|\left\langle e_{m}, h_{n}\right\rangle\right| \leq C_{k} e^{-k\left|n^{1 /(2 \alpha)}-m^{1 /(2 \alpha)}\right|}, m, n, k \in \mathbb{N} .
$$

Then the analysis operator $U_{E}$ is continuous from $\Sigma^{\alpha}$ into $\mathfrak{s}^{1 /(2 \alpha)}$, the synthesis operator $T_{E}$ is continuous from $\mathfrak{s}^{1 /(2 \alpha)}$ into $\Sigma^{\alpha}$, and the frame operator $T_{E} U_{E}$ is continuous from $\Sigma^{\alpha}$ into $\Sigma^{\alpha}$.

\section{Boundedness and Banach Frames Derived from Sub-exponential Localization of Frames}

In this section we extend statements from [23] for polynomially and exponentially localized frames to the case of sub-exponentially localized frames (Theorem 5.4 below). We will use the Jaffard's theorem [27] given there for the sub-exponential and exponential case (see Theorem 5.2 below).

First recall the Schur's test: If $\left(A_{m, n}\right)_{m, n \in \mathbb{N}}$ is an infinite matrix satisfying $\sup _{m \in \mathbb{N}} \sum_{n \in \mathbb{N}}\left|A_{m, n}\right| \leq K_{1}$ and $\sup _{n \in \mathbb{N}} \sum_{m \in \mathbb{N}}\left|A_{m, n}\right| \leq K_{2}$, then the corresponding matrix type operator $\mathcal{A}$ is well defined and bounded from $\ell^{p}$ into $\ell^{p}$ for $1 \leq p \leq \infty$ and the operator norm $\|\mathcal{A}\|_{\ell^{p} \rightarrow \ell^{p}} \leq K_{1}^{1 / p^{\prime}} K_{2}^{1 / p}$.

Let $\beta \in(0,1]$ and $\gamma \in(0, \infty)$. Define $\mathcal{E}_{\gamma, \beta}$ to be the space of matrices $\left(A_{m, n}\right)_{m, n \in \mathbb{N}}$ satisfying the following condition:

$$
\exists C_{\gamma} \in(0, \infty) \text { so that }\left|A_{m, n}\right| \leq C_{\gamma} e^{-\gamma|m-n|^{\beta}}, m, n \in \mathbb{N} .
$$

By the Schur's test, when $\left(A_{m, n}\right)_{m, n \in \mathbb{N}} \in \mathcal{E}_{\gamma, \beta}$, then the corresponding matrix type operator $\mathcal{A}$ is well defined and bounded from $\ell^{2}$ into $\ell^{2}$, and for the operator norm one has that $\|\mathcal{A}\|_{\ell^{2} \rightarrow \ell^{2}} \leq 2 C_{\gamma} P_{\gamma, \beta}$, where $C_{\gamma}$ is the constant from (13) and $P_{\gamma, \beta}$ denotes the sum of the convergent series $\sum_{j=0}^{\infty} e^{-\gamma j^{\beta}}$.

We also need the following statements, an extension of [23, Lemmas 2 and 3] with a sketch of a proof in the spirit of that paper. It should be noted that the statements can be traced back to [18] and [24, Secs. 2, 7]. ${ }^{1}$

Lemma 5.1 For every $\gamma \in(0, \infty)$ and $\beta \in(0,1]$, the following holds.

(a) There exists a positive number $C$ so that $\sum_{k \in \mathbb{N}} e^{-\gamma|m-k|^{\beta}} e^{-\gamma|k-n|^{\beta}} \leq$ $C e^{-(\gamma / 2)|m-n|^{\beta}}$ for every $m, n \in \mathbb{N}$.

\footnotetext{
1 We are thankful to the referee for pointing us to Refs. [18,24].
} 
(b) If the matrix $\left(A_{m, n}\right)_{m, n \in \mathbb{N}}$ belongs to $\mathcal{E}_{\gamma, \beta}$ and $\mu$ is a $\beta_{\mu}$-sub-exponential weight with $\beta_{\mu}<\beta$, then $\mathcal{A}$ maps boundedly $\ell_{\mu}^{p}\left(\mathbb{N}_{0}\right)$ into $\ell_{\mu}^{p}\left(\mathbb{N}_{0}\right)$ for every $p \in[1, \infty]$.

Proof (a) can be proved following the idea of [23, Lemma 2].

(b) Let $\gamma_{\mu}$ come from the $\beta_{\mu}$-sub-exponential weight $\mu$, i.e., $\mu(t+x) \leq$ $C_{\mu} e^{\gamma_{\mu}|t|^{\beta_{\mu}}} \mu(x), t, x \in \mathbb{R}$. This gives $\mu(x)=\mu(x-n+n) \leq C_{\mu} e^{\gamma_{\mu}|x-n|^{\beta_{\mu}}} \mu(n)$, $x, n \in \mathbb{N}$. Choose $k>\gamma \beta /\left(\gamma_{\mu} \beta_{\mu}\right)$. With this $k$ and the assumption $\beta_{\mu}<\beta$, one obtains that there is a constant $C_{1}>0$ so that

$$
e^{-(\gamma / k)|x-n|^{\beta}} \mu(n)^{-1} \mu(x) \leq C_{1}
$$

This follows from the fact that for the chosen $k$ the function $f(u)=\gamma_{\mu} u^{\beta_{\mu}}-$ $(\gamma / k) u^{\beta}, u \in[1, \infty)$, is bounded.

The rest of the proof can be done by trivial adaptations using [23, Lemma 3], (extending $k$ if it is necessary) and showing first that $\mathcal{A}$ maps boundedly $\ell_{\mu}^{p}\left(\mathbb{N}_{0}\right)$ into $\ell_{\mu}^{p}\left(\mathbb{N}_{0}\right)$ for $p=1$ and $p=\infty$ and then using interpolation.

Theorem 5.2 (Jaffard) Given $\beta \in(0,1]$ and $\gamma \in(0, \infty)$, let $\left(A_{m, n}\right)_{m, n \in \mathbb{N}} \in \mathcal{E}_{\gamma, \beta}$ and let the corresponding matrix type operator $\mathcal{A}$ be invertible on $\ell^{2}$. Then $\mathcal{A}^{-1} \in \mathcal{E}_{\gamma_{1}, \beta}$ for some $\gamma_{1} \in(0, \gamma)$.

In "Appendix" we will give a sketch of the Jaffard's proof.

Remark 5.3 The exponential localization type condition $\left|A_{m, n}\right| \leq C_{\gamma} e^{-\gamma|m-n|}$, $m, n \in \mathbb{N}$, considered in [23], implies that

$$
\forall \beta \in(0,1) \forall k \in \mathbb{N} \exists \widetilde{C_{k}} \text { so that }\left|A_{m, n}\right| \leq \widetilde{C_{k}} e^{-k|m-n|^{\beta}}, m, n \in \mathbb{N} \text {. }
$$

Here we consider the more general case intrinsically related to $\beta \in(0,1)$.

Theorem 5.4 Let $p \in[1, \infty)$ and $G$ be a Riesz basis for $\mathcal{H}$, and let $E$ be a frame for $\mathcal{H}$ which is $\beta$-sub-exponentially or exponentially localized (respectively, $(k+1+\varepsilon)$ localized for some $\varepsilon>0$ ) with respect to $G$. Let $\mu$ be $\beta_{\mu}$-sub-exponential weight and let $\beta_{\mu}<\beta$ in the case of $\beta$-sub-exponentially localized frame $E$ (respectively, let $\mu$ be a $k$-moderate weight) with $\mu(n) \geq 1$ for every $n \in \mathbb{N}$ and $\ell_{\mu}^{p} \subset \ell^{2}$.

Then for every $p \in[1, \infty)$ the following statements hold.

(i) The analysis operator $U_{E}$ maps boundedly $\mathcal{H}_{G}^{\ell_{\mu}^{p}}$ into $\ell_{\mu}^{p}$.

(ii) The synthesis operator $T_{E}$ maps boundedly $\ell_{\mu}^{p}$ into $\mathcal{H}_{G}^{\ell_{\mu}^{p}}$.

(iii) The frame operator $S_{E}=T_{E} U_{E}$ is invertible on $\mathcal{H}_{G}^{\ell_{\mu}^{p}}$ and the series in $S_{E} f=$ $\sum_{n=1}^{\infty}\left\langle f, e_{n}\right\rangle e_{n}$ converges unconditionally.

(iv) The canonical dual frame $\left(\tilde{e}_{n}\right)_{n=1}^{\infty}$ of $\left(e_{n}\right)_{n=1}^{\infty}$ has the same type of localization as $\left(e_{n}\right)_{n=1}^{\infty}$, i.e., it is $\beta$-sub-exponentially or exponentially localized (resp. $(k+1+\varepsilon)$ localized) with respect to $G$.

(v) The frame expansions $f=\sum_{n=1}^{\infty}\left\langle f, e_{n}\right\rangle \widetilde{e_{n}}=\sum_{n=1}^{\infty}\left\langle f, \widetilde{e_{n}}\right\rangle e_{n}$ hold with unconditional convergence in $\mathcal{H}_{G}^{\ell_{\mu}^{p}}$. 
(vi) There is norm equivalence between $\|f\|_{\mathcal{H}_{G}^{\ell_{\mu}^{p}}}$, $\left\|\mid\left(\left\langle f, e_{n}\right\rangle\right)_{n=1}^{\infty}\right\| \|_{\ell_{\mu}^{p}}$, and $\left\|\left|\left(\left\langle f, \tilde{e_{n}}\right\rangle\right)_{n=1}^{\infty}\right|\right\|_{\ell_{\mu}^{p}}$.

Proof In the cases of polynomial and exponential localization, the assertions are given in [23, Prop. 8 and Prop. 10]. For the sub-exponential case, one can proceed in a similar way, but using Lemma 5.1 and Theorem 5.2. For the sake of completeness, we sketch a proof.

Let $\gamma>0$ and $C>0$ come from the sub-exponential localization of $E$ with respect to $G$, i.e., $\max \left\{\left|\left\langle e_{m}, g_{n}\right\rangle\right|,\left|\left\langle e_{m}, \widetilde{g_{n}}\right\rangle\right|\right\} \leq C \mathrm{e}^{-\gamma|m-n|^{\beta}}, m, n \in \mathbb{N}$. Consider the matrix $\left(A_{m, n}\right)_{m, n \in \mathbb{N}}$ determined by $A_{m, n}=e^{-\gamma|m-n|^{\beta}}$ for $m, n \in \mathbb{N}$.

(i) Let $f \in \mathcal{H}_{G}^{\ell_{\mu}^{p}}$ and thus $\left(\left|\left\langle f, \tilde{g}_{n}\right\rangle\right|\right)_{n=1}^{\infty} \in \ell_{\mu}^{p}$. By Lemma 5.1(b), we have that $\mathcal{A}\left(\left|\left\langle f, \tilde{g}_{n}\right\rangle\right|\right)_{n=1}^{\infty}$ belongs to $\ell_{\mu}^{p}$. Furthermore, for $m \in \mathbb{N}$ we have

$$
\left|\left\langle f, e_{m}\right\rangle\right| \leq \sum_{n=1}^{\infty}\left|\left\langle f, \tilde{g_{n}}\right\rangle\left\langle g_{n}, e_{m}\right\rangle\right| \leq C \sum_{n=1}^{\infty} A_{m, n}\left|\left\langle f, \tilde{g_{n}}\right\rangle\right|
$$

Therefore, $\left(\left\langle f, e_{m}\right\rangle\right)_{m=1}^{\infty}$ also belongs to $\ell_{\mu}^{p}$ and

$$
\left\|\left(\left\langle f, e_{m}\right\rangle\right)_{m=1}^{\infty}\right\|_{\ell_{\mu}^{p}} \leq C\left\|\mathcal{A}\left(\left|\left\langle f, \tilde{g}_{n}\right\rangle\right|\right)_{n=1}^{\infty}\right\|_{\ell_{\mu}^{p}} \leq C\|\mathcal{A}\| \cdot\|f\|_{\mathcal{H}_{G}^{\ell_{\mu}^{p}}}
$$

(ii) Let $c=\left(c_{n}\right)_{n=1}^{\infty} \in \ell_{\mu}^{p}\left(\subseteq \ell^{2}\right)$. Then the series $\sum_{n=1}^{\infty} c_{n} e_{n}$ converges in $\mathcal{H}$ and let us denote its sum by $y$. Since $\mathcal{A}\left(\left|c_{n}\right|\right)_{n=1}^{\infty} \in \ell_{\mu}^{p}$ by Lemma 5.1(b), and since $\left|\left\langle y, \widetilde{g}_{m}\right\rangle\right| \leq C \sum_{n=1}^{\infty}\left|c_{n}\right|\left|\left\langle e_{n}, \widetilde{g}_{m}\right\rangle\right| \leq C \sum_{n=1}^{\infty} A_{m, n}\left|c_{n}\right|$ for every $m \in \mathbb{N}$, it follows that $U_{\widetilde{G}} y \in \ell_{\mu}^{p}$ and therefore the element $T_{G} U_{\widetilde{G}} y=y$ belongs to $\mathcal{H}_{G}^{\ell_{\mu}^{p}}$. Hence, $T_{E}$ maps $\ell_{\mu}^{p}$ into $\mathcal{H}_{G}^{\ell_{\mu}^{p}}$ and furthermore, $\left\|T_{E} c\right\|_{\mathcal{H}_{G}^{\ell_{\mu}^{p}}}=$ $\|y\|_{\mathcal{H}_{G}^{\ell_{\mu}^{p}}}=\left\|U_{\widetilde{G}} y\right\|_{\ell_{\mu}^{p}} \leq C\left\|\mathcal{A}\left(\left|c_{n}\right|\right)\right\|_{\ell_{\mu}^{p}}$, which by Lemma 5.1 implies that $\left\|T_{E} c\right\|_{\mathcal{H}_{G}^{\ell_{\mu}^{p}}} \leq C\|\mathcal{A}\|\|c\|_{\ell_{\mu}^{p}}$.

(iii) By (i) and (ii), $S_{E}$ maps boundedly $\mathcal{H}_{G}^{\ell_{\mu}^{p}}$ into $\mathcal{H}_{G}^{\ell_{\mu}^{p}}$. For the unconditional convergence, take any re-ordering $N_{1}$ of $\mathbb{N}$. Let $f \in \mathcal{H}_{G}^{\ell_{\mu}^{p}}$. Consider $\sum_{n \in N_{1}}\left\langle f, e_{n}\right\rangle e_{n}$ and take $\varepsilon>0$. Since $\left(\left\langle f, e_{n}\right\rangle\right)_{n \in N_{1}} \in \ell_{\mu}^{p}$, there is a finite set $N_{2} \subset N_{1}$ so that $\left\|\left(\left\langle f, e_{n}\right\rangle\right)_{n \in N_{1} \backslash N_{2}}\right\|_{\ell_{\mu}^{p}}<\varepsilon$. Then, for every finite $N_{3}$ such that $N_{3} \supset N_{2}$, $N_{3} \subset N_{1}$, one has that $\left\|S f-\sum_{n \in N_{3}}\left\langle f, e_{n}\right\rangle e_{n}\right\| \leq\left\|T_{E}\right\|\left\|\left(\left\langle f, e_{n}\right\rangle\right)_{n \in N_{1} \backslash N_{2}}\right\|_{\ell_{\mu}^{p}}<$ $\left\|T_{E}\right\| \varepsilon$. Therefore, $\sum_{n \in N_{1}}\left\langle f, e_{n}\right\rangle e_{n}$ converges to $S f$.

Finally, let us show the bijectivity of $S_{E}$ on $\mathcal{H}_{G}^{\ell_{\mu}^{p}}$. Consider the operator $\mathcal{V}:=U_{\widetilde{G}} S_{E} T_{G}$ and observe that it is invertible on $\ell^{2}$ and it maps boundedly $\ell_{\mu}^{p}$ into $\ell_{\mu}^{p}$. Let $\left(V_{m, n}\right)_{m, n \in \mathbb{N}}$ be the corresponding matrix of $\mathcal{V}$. Since $\left|V_{m, n}\right|=\left|\left\langle S_{E} g_{n}, \widetilde{g_{m}}\right\rangle\right| \leq \sum_{i}\left|\left\langle g_{n}, e_{i}\right\rangle\right| \cdot\left|\left\langle e_{i}, \widetilde{g_{m}}\right\rangle\right|$, by Lemma 5.1(a), there is a positive constant $C_{1}$ so that $\left|V_{m, n}\right| \leq C_{1} e^{-(\gamma / 2)|m-n|^{\beta}}$. Now by Theorem 5.2 it follows that $\mathcal{V}^{-1} \in \mathcal{E}_{\gamma_{1}, \beta}$ for some $\gamma_{1} \in(0, \gamma / 2)$. By Lemma 5.1, it follows that 
$\mathcal{V}^{-1}$ maps boundedly $\ell_{\mu}^{p}$ into $\ell_{\mu}^{p}$. Therefore, $\mathcal{V}$ is a bounded bijection of $\ell_{\mu}^{p}$ onto $\ell_{\mu}^{p}$. Now the representation $S_{E}=T_{G} \mathcal{V} U_{\widetilde{G}}$ implies that $S_{E}$ is a bounded bijection of $\mathcal{H}_{G}^{\ell_{\mu}^{p}}$ onto $\mathcal{H}_{G}^{\ell_{\mu}^{p}}$.

(iv) Use the operator $\mathcal{V}$, determined in (iii), and observe that for $m, n \in \mathbb{N}$ we have $\left\langle\widetilde{e_{m}}, g_{n}\right\rangle=\sum_{j=1}^{\infty}\left\langle e_{m}, g_{j}\right\rangle \overline{\left(V^{-1}\right)_{j n}}$ and $\left\langle\widetilde{e_{m}}, \widetilde{g_{n}}\right\rangle=\sum_{j=1}^{\infty}\left\langle e_{m}, \widetilde{g_{j}}\right\rangle \overline{\left(V^{-1}\right)_{j n}}$. Since $\mathcal{V}^{-1} \in \mathcal{E}_{\gamma_{1}, \beta}$ for some $\gamma_{1} \in(0, \gamma / 2)$, one can apply Lemma 5.1(a) appropriately to conclude.

(v) follows from (iii) and for (vi) one can use the representations $f=S_{E}^{-1} S_{E}=$ $S_{\widetilde{E}^{-1}} S_{\widetilde{E}} f$ and the already proved (i)-(iv).

\section{Expansions in Fréchet Spaces Via Localized Frames}

Our goal is expansion of elements of a Fréchet space and its dual via localized frames and coefficients in a corresponding Fréchet sequence space. First we present in the next theorem general results related to frames localized with respect to a Riesz basis. In the next section, we will apply this theorem using frames localized with respect to the Hermite orthonormal basis in order to obtain frame expansions in the spaces $\mathcal{S}$ and $\Sigma^{\alpha}, \alpha>1 / 2$, and their duals. To clarify notation, for an element $e \in \mathcal{H}$, its corresponding element in $\mathcal{H}^{*}$ by the Rieszs representation theorem will be denoted by the bold-style letter e. Note that in the setting of Lemma 2.2, for an element $e$ from $X_{0}$, one can conclude that e belongs to $X_{0}^{*}$; thus, for $e \in X_{F}\left(\subseteq X_{0}\right)$, we can consider e as an element of $X_{F}^{*}$.

Theorem 6.1 Let $G$ be a Riesz basis for $\mathcal{H}, k \in \mathbb{N}_{0}$, and $\mu_{k}$ be a $\beta_{k}$-sub-exponential (resp. $k$-moderate) weight so that (5) holds. Let the spaces $\Theta_{k}$ and $X_{k}$ be as in Lemma 2.2. Assume that $E=\left(e_{n}\right)_{n=1}^{\infty}$ is a sequence with elements in $X_{F}$ forming a frame for $\mathcal{H}$ which is $\beta$-sub-exponentially localized with $\beta>\beta_{k}$ for all $k \in \mathbb{N}_{0}$ or exponentially localized (respectively, $s$-localized for every $s \in \mathbb{N}$ ) with respect to $G$. Then, $\widetilde{e}_{n} \in X_{F}$, $n \in \mathbb{N}$, and the following statements hold:

(i) The analysis operator $U_{E}$ is $F$-bounded from $X_{F}$ into $\Theta_{F}$, the synthesis operator $T_{E}$ is $F$-bounded from $\Theta_{F}$ into $X_{F}$, and the frame operator $S_{E}$ is $F$-bounded and bijective from $X_{F}$ onto $X_{F}$ with unconditional convergence of the series in $S_{E} f=\sum_{n=1}^{\infty}\left\langle f, e_{n}\right\rangle e_{n}$.

(ii) For every $f \in X_{F}$,

$$
f=\sum_{n=1}^{\infty}\left\langle f, \widetilde{e_{n}}\right\rangle e_{n}=\sum_{n=1}^{\infty}\left\langle f, e_{n}\right\rangle \widetilde{e_{n}}\left(\text { with convergence in } X_{F}\right)
$$

with $\left(\left\langle f,{\tilde{e_{n}}}\right\rangle\right)_{n=1}^{\infty} \in \Theta_{F}$ and $\left(\left\langle f, e_{n}\right\rangle\right)_{n=1}^{\infty} \in \Theta_{F}$.

(iii) If $X_{F}$ and $\Theta_{F}$ have the following property with respect to $\left(g_{n}\right)_{n=1}^{\infty}$ :

$$
\mathcal{P}_{\left(g_{n}\right)}: \text { For } f \in \mathcal{H} \text {, one has } f \in X_{F} \text { if and only if }\left(\left\langle f, g_{n}\right\rangle\right)_{n=1}^{\infty} \in \Theta_{F} \text {. }
$$

then $X_{F}$ and $\Theta_{F}$ also have the properties $\mathcal{P}_{\left(e_{n}\right)}$ and $\mathcal{P}_{\left(\tilde{e}_{n}\right)}$. 
(iv) Both sequences $\left(\boldsymbol{e}_{n}\right)_{n=1}^{\infty}$ and $\left(\tilde{\boldsymbol{e}_{n}}\right)_{n=1}^{\infty}$ form Fréchet frames for $X_{F}$ with respect to $\Theta_{F}$.

(v) For every $g \in X_{F}^{*}$,

$$
g=\sum_{n=1}^{\infty} g\left(e_{n}\right) \tilde{\boldsymbol{e}_{n}}=\sum_{n=1}^{\infty} g\left(\tilde{e_{n}}\right) \boldsymbol{e}_{n}\left(\text { with convergence in } X_{F}^{*}\right)
$$

with $\left(g\left(e_{n}\right)\right)_{n=1}^{\infty} \in \Theta_{F}^{*}$ and $\left(g\left(\tilde{e_{n}}\right)\right)_{n=1}^{\infty} \in \Theta_{F}^{*}$.

(vi) If $\left(a_{n}\right)_{n=1}^{\infty} \in \Theta_{F}^{*}$, then $\sum_{n=1}^{\infty} a_{n} \boldsymbol{e}_{n}$ (resp. $\sum_{n=1}^{\infty} a_{n} \widetilde{\boldsymbol{e}_{n}}$ ) converges in $X_{F}^{*}$, i.e., the mapping $f \mapsto \sum_{n=1}^{\infty}\left\langle f, e_{n}\right\rangle a_{n}$ (resp. $f \mapsto \sum_{n=1}^{\infty}\left\langle f, \tilde{e}_{n}\right\rangle a_{n}$ ) determines $a$ continuous linear functional on $X_{F}$.

Proof (i) The properties for $U_{E}, T_{E}$, and $S_{E}$ follow easily using Theorem 5.4(i)-(iii). Further, the bijectivity of $S_{E}$ on $X_{F}$ implies that $\tilde{e_{n}} \in X_{F}$ for every $n \in \mathbb{N}$.

(ii) By Theorem 5.4(v), for every $k \in \mathbb{N}$ and every $f \in X_{k}$ we have that $f=$ $\sum_{n=1}^{\infty}\left\langle f, \tilde{e_{n}}\right\rangle e_{n}=\sum_{n=1}^{\infty}\left\langle f, e_{n}\right\rangle \tilde{e_{n}}$ with convergence in $X_{k}$. This implies that for every $f \in X_{F}$, one has that $f=\sum_{n=1}^{\infty}\left\langle f, \tilde{e_{n}}\right\rangle e_{n}=\sum_{n=1}^{\infty}\left\langle f, e_{n}\right\rangle \widetilde{e_{n}}$ with convergence in $X_{F}$.

For every $k \in \mathbb{N}$ and every $f \in X_{k}$, by Theorem 5.4(i), we have that $\left(\left\langle f, e_{n}\right\rangle\right)_{n=1}^{\infty} \in \Theta_{k}$. Therefore, $\left(\left\langle f, e_{n}\right\rangle\right)_{n=1}^{\infty} \in \Theta_{F}$ for every $f \in X_{F}$. Furthermore, by Theorem 5.4(iv), $\left(\tilde{e_{n}}\right)_{n=1}^{\infty}$ has the same type of localization with respect to $G$ as $\left(e_{n}\right)_{n=1}^{\infty}$. Thus, applying Theorem 5.4(i) with $\left(\tilde{e_{n}}\right)_{n=1}^{\infty}$ as a starting frame, we get that $\left(\left\langle f, \tilde{e}_{n}\right\rangle\right)_{n=1}^{\infty} \in \Theta_{F}$ for $f \in X_{F}$.

(iii) If $f \in X_{F}$, it is already proved in (i) that $\left(\left\langle f, e_{n}\right\rangle\right)_{n=1}^{\infty} \in \Theta_{F}$ and $\left(\left\langle f, \tilde{e_{n}}\right\rangle\right)_{n=1}^{\infty} \in$ $\Theta_{F}$. To complete the proof of $\mathcal{P}_{\left(e_{n}\right)}$, assume that $f \in \mathcal{H}$ is such that $\left(\left\langle f, e_{n}\right\rangle\right)_{n=1}^{\infty} \in$ $\Theta_{F}$. Consider

$$
\left(\left\langle f, g_{n}\right\rangle\right)_{n=1}^{\infty}=\left(\left\langle f, \sum_{j=1}^{\infty}\left\langle g_{n}, \widetilde{e_{j}}\right\rangle e_{j}\right\rangle\right)_{n=1}^{\infty}=\left(\sum_{j=1}^{\infty}\left\langle\widetilde{e_{j}}, g_{n}\right\rangle\left\langle f, e_{j}\right\rangle\right)_{n=1}^{\infty}
$$

Let $k \in \mathbb{N}$. Since $\left(\left\langle f, e_{j}\right\rangle\right)_{j=1}^{\infty} \in \ell_{\mu_{k}}^{2}$ and by Theorem 5.4(iv), $\left(\tilde{e}_{n}\right)_{n=1}^{\infty}$ has the same type of localization with respect to $G$ as $\left(e_{n}\right)_{n=1}^{\infty}$, it follows from Lemma 5.1(b) (for the case of sub-exponential localization) and from the way of the proof of [23, Lemma 3] (for the case of polynomial and exponential localization) that $\left(\sum_{j=1}^{\infty}\left\langle\tilde{e_{j}}, g_{n}\right\rangle\left\langle f, e_{j}\right\rangle\right)_{n=1}^{\infty} \in \ell_{\mu_{k}}^{2}$. Therefore, $\left(\left\langle f, g_{n}\right\rangle\right)_{n=1}^{\infty} \in \Theta_{F}$ and thus, by $\mathcal{P}_{\left(g_{n}\right)}$, it follows that $f \in X_{F}$. For completing the proof of $\mathcal{P}_{\left(\widetilde{e_{n}}\right)}$, if $f \in \mathcal{H}$ is such that $\left(\left\langle f, \tilde{e_{n}}\right\rangle\right)_{n=1}^{\infty} \in \Theta_{F}$, it follows in a similar way as above that $f \in X_{F}$.

(iv) By (i), $\left(\mathbf{e}_{n}(f)\right)_{n=1}^{\infty} \in \Theta_{F}$ for $f \in X_{F}$, and by Theorem 5.4(vi), for $k \in \mathbb{N}$ and $f \in X_{k}$, the norms $\left\|\mid\left(\left\langle f, e_{n}\right\rangle\right)_{n=1}^{\infty}\right\|_{\Theta_{k}}$ and $\|f\|_{X_{k}}$ are equivalent. Furthermore, it follows from Theorem 5.4 that the operator $V:=\left.S_{E}^{-1} T_{E}\right|_{\Theta_{F}}$ maps $\Theta_{F}$ into $X_{F}$ and it is $F$-bounded. Clearly, $V\left(\mathbf{e}_{n}(f)\right)_{n=1}^{\infty}=f, f \in X_{F}$. Therefore, $\left(\mathbf{e}_{n}\right)_{n=1}^{\infty}$ is an $F$-frame for $X_{F}$ with respect to $\Theta_{F}$. In an analogue way, $\left(\widetilde{\mathbf{e}_{n}}\right)_{n=1}^{\infty}$ is also an $F$-frame for $X_{F}$ with respect to $\Theta_{F}$. 
(v) The representations in (i) can be re-written as $f=\sum_{n=1}^{\infty} \widetilde{\mathbf{e}}_{n}(f) e_{n}=$ $\sum_{n=1}^{\infty} \mathbf{e}_{n}(f) \tilde{e_{n}}, f \in X_{F}$, which implies validity of (16) for $g \in X_{F}^{*}$.

For the rest of the proof, consider the $F$-bounded (and hence continuous) operator $V$ from the proof of (iv) and observe that $\tilde{e_{n}}=V \delta_{n}, n \in \mathbb{N}$. This implies that for $g \in X_{F}^{*}$ we have $\left(g\left(\widetilde{e_{n}}\right)\right)_{n=1}^{\infty}=\left(g V\left(\delta_{n}\right)\right)_{n=1}^{\infty} \in \Theta_{F}^{\circledast}$. With similar arguments, considering the operator $\widetilde{V}=\left.S_{\widetilde{E}}^{-1} T_{\widetilde{E}}\right|_{\Theta_{F}}$, it follows that $\left(g\left(e_{n}\right)\right)_{n=1}^{\infty} \in \Theta_{F}^{\circledast}$.

(vi) Let $\left(a_{n}\right)_{n=1}^{\infty} \in \Theta_{F}^{*}$ and thus there is $k_{0} \in \mathbb{N}$ so that $\left(a_{n}\right)_{n=1}^{\infty} \in \Theta_{k_{0}}^{*}$, i.e., $C:=$ $\sum_{n=1}^{\infty}\left|a_{n}\right|^{2}\left|\mu_{k_{0}}(n)\right|^{-2}<\infty$. By Theorem 5.4(vi), there is a positive constant $B_{k_{0}}$ so that $\left\|\mid\left(\left\langle f, e_{n}\right\rangle\right)_{n=1}^{\infty}\right\|\left\|_{\Theta_{k_{0}}} \leq B_{k_{0}}\right\| f \|_{X_{k_{0}}}$ for every $f \in X_{F}$. Let $f \in X_{F}$. By (i), $\left(\left\langle f, e_{n}\right\rangle\right)_{n=1}^{\infty} \in \Theta_{F}$. Therefore, $\sum_{n=1}^{\infty}\left\langle f, e_{n}\right\rangle a_{n}$ converges and furthermore,

$$
\begin{aligned}
\left|\sum_{n=1}^{\infty}\left\langle f, e_{n}\right\rangle a_{n}\right|^{2} & \leq\left(\sum_{n=1}^{\infty}\left|\left\langle f, e_{n}\right\rangle\right|^{2}\left|\mu_{k_{0}}(n)\right|^{2}\right)\left(\sum_{n=1}^{\infty}\left|a_{n}\right|^{2}\left|\mu_{k_{0}}(n)\right|^{-2}\right) \\
& =C\left\|\mid\left(\left\langle f, e_{n}\right\rangle\right)_{n=1}^{\infty}\right\|_{\Theta_{k_{0}}} \leq B_{k_{0}} C\|f\|_{X_{k_{0}}},
\end{aligned}
$$

which implies continuity of the linear mapping $f \mapsto \sum_{n=1}^{\infty}\left\langle f, e_{n}\right\rangle a_{n}$. In a similar way, it follows that $f \mapsto \sum_{n=1}^{\infty}\left\langle f, \widetilde{e_{n}}\right\rangle a_{n}$ determines a continuous linear functional on $X_{F}$.

Remark 6.2 Note that in the setting of the above theorem, when $G$ is an orthonormal basis of $\mathcal{H}$ or more generally, when $G$ is a Riesz basis for $\mathcal{H}$ satisfying any of the following two conditions:

$\left(\mathcal{P}_{1}\right): \forall s \in \mathbb{N} \exists C_{s}>0:\left|\left\langle g_{m}, g_{n}\right\rangle\right| \leq C_{s}(1+|m-n|)^{-s}, m, n \in \mathbb{N}$, $\left(\mathcal{P}_{2}\right): \exists s>0 \exists C_{s}>0:\left|\left\langle g_{m}, g_{n}\right\rangle\right| \leq C_{s} \mathrm{e}^{-s|m-n|}, m, n \in \mathbb{N}$,

then the property $\mathcal{P}_{\left(g_{n}\right)}$ is satisfied.

\subsection{Frame Expansions of Tempered Distributions and Ultradistributions}

Here we apply Theorem 6.1 to obtain series expansions in the spaces $\mathcal{S}$ and $\Sigma^{\alpha}$ $(\alpha>1 / 2)$, and their dual spaces, via frames which are localized with respect to the Hermite basis.

Theorem 6.3 Assume that the sequence $\left(e_{n}\right)_{n=1}^{\infty}$ with elements from $\mathcal{S}(\mathbb{R})\left(\right.$ resp. in $\left.\Sigma^{\alpha}\right)$ is a frame for $L^{2}(\mathbb{R})$ which is polynomially (resp. sub-exponentially or exponentially) localized with respect to the Hermite basis $\left(h_{n}\right)_{n=1}^{\infty}$ with decay $\gamma$ for every $\gamma \in \mathbb{N}$. Let $\left(g_{n}\right)_{n=1}^{\infty}=\left(h_{n}\right)_{n=1}^{\infty}$. Then $\mathcal{P}_{\left(g_{n}\right)}$ and the conclusions in Theorem 6.1 hold with $X_{F}$ replaced by $\mathcal{S}\left(\right.$ resp. $\left.\Sigma^{\alpha}\right)$ and $\Theta_{F}$ replaced by $\mathbf{s}\left(\right.$ resp. $\left.\mathfrak{s}^{1 /(2 \alpha)}\right)$.

Proof For $k \in \mathbb{N}_{0}$, consider the $k$-moderate weight $\mu_{k}(x)=(1+|x|)^{k}$. The spaces $\Theta_{k}:=\ell_{\mu_{k}}^{2}, k \in \mathbb{N}_{0}$, satisfy (1)-(2) and their projective limit $\Theta_{F}$ is the space $\mathbf{s}$. Consider the spaces $X_{k}:=\mathcal{H}_{\left(h_{n}\right)}^{\Theta_{k}}, k \in \mathbb{N}_{0}$, which satisfy (1)-(2). As observed after Theorem 6.1, the property $\mathcal{P}_{\left(h_{n}\right)}$ is satisfied. Since for $f \in L^{2}(\mathbb{R})$ one has that $f \in \mathcal{S}$ if and only if $\left(\left\langle f, h_{n}\right\rangle_{n=1}^{\infty}\right) \in \mathbf{s}$, it now follows that $X_{F}=\mathcal{S}$. Then the conclusions of Theorem 6.3 follow from Theorem 6.1. 
The respective part of the theorem follows in a similar way.

As noticed in [35], having in mind the known expansions of tempered distributions $\left(\mathcal{S}\left(\mathbb{R}_{+}\right)\right)^{\prime}[16,38]$ and Beurling ultradistributions $\left(G_{\alpha}^{\alpha}\left(\mathbb{R}_{+}\right)\right)^{\prime}[17,25,26]$, and their test spaces, by the use of the Laguerre orthonormal basis $l_{n}, n \in \mathbb{N}$, and validity of the corresponding properties $\mathcal{P}_{\left(l_{n}\right)}$, we can transfer the above results to the mentioned classes of distributions and ultradistributions over $\mathbb{R}_{+}$.

Remark 6.4 For Proposition 4.1 (resp. 4.3), it is of interest to consider cases when $X_{F}$ is the space $\mathcal{S}$ (resp. $\Sigma^{\alpha}$ ). This was the case in Corollary 4.2 (resp. Corollary 4.4) taking $G$ to be the Hermite basis. Based on Theorem 6.3, we can determine further cases. If $G$ is a frame for $L^{2}(\mathbb{R})$ with elements from $\mathcal{S}$ and polynomially localized with respect to $\left(h_{n}\right)_{n=1}^{\infty}$, then one also has $X_{F}=\mathcal{S}$. Indeed, under such assumptions on $G$, Theorem 6.3 implies that $f \in \mathcal{S}$ if and only if $\left(\left\langle f, \tilde{g}_{n}\right\rangle\right) \in \mathbf{s}$, and by the construction of $X_{F}$, one has that $f \in X_{F}$ if and only if $\left(\left\langle f, \tilde{g_{n}}\right\rangle\right) \in \mathbf{s}$.

Concerning Proposition 4.3, with $\alpha>1 / 2$ and $\beta=1 /(2 \alpha)$, we have a similar conclusion for $\Sigma^{\alpha}$.

Example 6.5 As an illustration of Theorems 6.1 and 6.3, we give the next example. Let $r \in \mathbb{N}$ and for $i=1,2, \ldots, r$, take $\varepsilon_{i} \geq 0$ and a sequence $\left(a_{n}^{i}\right)_{n=1}^{\infty}$ of complex numbers satisfying $\left|a_{n}^{i}\right| \leq \varepsilon_{i}$ for $n \geq 2, \sum_{i=1}^{r}\left|a_{1}^{i}\right| \leq 1$, and $\sum_{i=1}^{r} \varepsilon_{i}<1$. For $n \in \mathbb{N}$, consider $e_{n}:=h_{n}+\sum_{i=1}^{r} a_{n}^{i} h_{n+i}$. The sequence $\left(e_{n}\right)_{n=1}^{\infty}$ is a Riesz basis for $L^{2}(\mathbb{R})$ and it is $s$-localized with respect to the Hermite orthonormal basis $\left(h_{n}\right)_{n=1}^{\infty}$ for every $s>0$, as well as exponentially localized with respect to $\left(h_{n}\right)_{n=1}^{\infty}$. In order to show that $\left(e_{n}\right)_{n=1}^{\infty}$ is a Riesz basis for $L^{2}(\mathbb{R})$, we will represent $\left(e_{n}\right)_{n=1}^{\infty}$ as a sequence $\left(U h_{n}\right)_{n=1}^{\infty}$ for some bounded bijective operator from $L^{2}(\mathbb{R})$ onto $L^{2}(\mathbb{R})$ using similar techniques as in [7, Example 1]. Define $U$ by $U h_{n}:=h_{n}+\sum_{i=1}^{r} a_{n}^{i} h_{n+i}, n \in \mathbb{N}$, and by linearity on the linear span of $h_{n}, n \in \mathbb{N}$. The obtained operator is bounded on the linear span, so extend it by continuity on $L^{2}(\mathbb{R})$, leading to a bounded operator on $L^{2}(\mathbb{R})$. It remains to show the bijectivity of $U$. Let $f \in L^{2}(\mathbb{R})$. Then

$$
U f-\left\langle f, h_{1}\right\rangle h_{1}=\sum_{n=2}^{\infty}\left\langle f, h_{n}\right\rangle h_{n}+\sum_{n=1}^{\infty} \sum_{i=1}^{r} a_{n}^{i}\left\langle f, h_{n}\right\rangle h_{n+i} \in \overline{\operatorname{span}}\left\{h_{n}\right\}_{n=2}^{\infty}
$$

and thus, $\|U f\| \geq\left|\left\langle f, h_{1}\right\rangle\right|$. Furthermore, for $f \in L^{2}(\mathbb{R})$,

$$
U f=\sum_{n=1}^{\infty}\left\langle f, h_{n}\right\rangle U h_{n}=f+\sum_{n=1}^{\infty} \sum_{i=1}^{r} a_{n}^{i}\left\langle f, h_{n}\right\rangle h_{n+i},
$$

leading to

$$
\begin{aligned}
\|U f\| & \leq\|f\|+\sum_{i=1}^{r}\left\|\sum_{n=1}^{\infty} a_{n}^{i}\left\langle f, h_{n}\right\rangle h_{n+i}\right\|=\|f\|+\sum_{i=1}^{r}\left\|\left(a_{n}^{i}\left\langle f, h_{n}\right\rangle\right)_{n=1}^{\infty}\right\|_{\ell^{2}} \\
& \left.\leq\|f\|+\sum_{i=1}^{r} \sqrt{\left|a_{1}^{i}\right|^{2}\|f\|^{2}+\varepsilon_{i}^{2}\|f\|^{2}} \leq\|f\|+\sum_{i=1}^{r}\left(\left|a_{1}^{i}\right|+\varepsilon_{i}\right)\|f\|\right)
\end{aligned}
$$




$$
\begin{aligned}
& \leq 3\|f\|, \\
\|U f-f\| & \leq \sum_{i=1}^{r} \sqrt{\left|a_{1}^{i}\right|^{2}\left|\left\langle f, h_{1}\right\rangle\right|^{2}+\sum_{n=2}^{\infty}\left|a_{n}^{i}\right|^{2}\left|\left\langle f, h_{n}\right\rangle\right|^{2}} \\
& \leq\|U f\|+\left(\sum_{i=1}^{r} \varepsilon_{i}\right)\|f\| \\
& \leq \frac{3+\sum_{i=1}^{r} \varepsilon_{i}}{4}\|U f\|+\frac{3+\sum_{i=1}^{r} \varepsilon_{i}}{4}\|f\| .
\end{aligned}
$$

Since $\left(3+\sum_{i=1}^{r} \varepsilon_{i}\right) / 4<1$, it follows from [7, Lemma 1] that the bounded operator $U$ is bijective on $L^{2}(\mathbb{R})$ and thus $\left(e_{n}\right)_{n=1}^{\infty}$ is a Riesz basis for $L^{2}(\mathbb{R})$.

Note that under the assumptions of the example, the classical way to obtain invertibility of $U$ does not apply, because $\|U-I d\|$ is not necessarily smaller than 1 .

Remark 6.6 As explained in [23], when dealing with Gabor frames and localization, the natural bases to be considered in this respect are the Wilson bases, but then Gabor frames are not localized with respect to a Wilson basis in the strict sense of the definition of polynomial and exponential localization. However, under appropriate conditions, the authors of [23] still obtain statements in the spirit of Theorem 5.4 which now leads to conclusions as in Theorem 6.1.

Acknowledgements The authors acknowledge support from OeAD GmbH through the Scientific and Technological Cooperation Projects MULT_DR 01/2017 and SRB 01/2018, from the Vienna Science and Technology Fund (WWTF) through Project VRG12-009, from the Serbian Academy of Sciences and Arts, project F10, and from the Austrian Science Fund (FWF) through the project "FLAME" Y 551-N13. The second author is grateful for the hospitality of the University of Novi Sad, where most of the research on the presented topic was done.

Open Access This article is licensed under a Creative Commons Attribution 4.0 International License, which permits use, sharing, adaptation, distribution and reproduction in any medium or format, as long as you give appropriate credit to the original author(s) and the source, provide a link to the Creative Commons licence, and indicate if changes were made. The images or other third party material in this article are included in the article's Creative Commons licence, unless indicated otherwise in a credit line to the material. If material is not included in the article's Creative Commons licence and your intended use is not permitted by statutory regulation or exceeds the permitted use, you will need to obtain permission directly from the copyright holder. To view a copy of this licence, visit http://creativecommons.org/licenses/by/4.0/.

\section{Appendix}

We add in Jaffard's proof some comments in the end. We also give a simple known assertions for the class $\mathcal{E}_{\gamma, \beta}$ defined on page 12.

Lemma 7.1 Let $\mathcal{A} \in \mathcal{E}_{\gamma, \beta}$.

(i) If $B$ belongs to $\mathcal{E}_{\gamma^{\prime}, \beta}$ for some $\gamma^{\prime} \in(0, \gamma)$, then $A B$ belongs to $\mathcal{E}_{\gamma^{\prime}, \beta}$.

(ii) If $B$ belongs to $\mathcal{E}_{\gamma, \beta}$, then $A B$ belongs to $\mathcal{E}_{\gamma^{\prime}, \beta}$ for every $\gamma^{\prime} \in(0, \gamma)$.

Proof (i) Under the assumptions, there exist positive constants $C_{A}$ and $C_{B}$ so that $\left|A_{m, n}\right| \leq C_{A} e^{-\gamma|m-n|^{\beta}}$ and $\left|B_{m, n}\right| \leq C_{B} e^{-\gamma^{\prime}|m-n|^{\beta}}$ for $m, n \in \mathbb{N}$. Further, using 


$$
\begin{aligned}
& |m-k|^{\beta}+|k-n|^{\beta} \geq|m-n|^{\beta}, \text { for every } m, n \in \mathbb{N}, \text { one has } \\
& \left|(A B)_{m, n}\right| \leq C_{A} C_{B} \sum_{k=1}^{\infty} e^{-\gamma^{\prime}|m-k|^{\beta}} e^{-\gamma|k-n|^{\beta}} \leq C_{A} C_{B} e^{-\gamma^{\prime}|m-n|^{\beta}} 2 \sum_{j=0}^{\infty} e^{-\left(\gamma-\gamma^{\prime}\right) j^{\beta}} .
\end{aligned}
$$

(ii) follows from (i).

Now we give some details for the Jaffard's proof of Theorem 5.2, providing explicit estimates for the bounds.

As in [27], $A A^{*}$ belongs to $\mathcal{E}_{\gamma^{\prime}, \beta}$ for $\gamma^{\prime} \in(0, \gamma)$ by Lemma 7.1, $A A^{*}=$ $\left\|A A^{*}\right\|(I d-R)$ for some operator $R$ with $\|R\|=r<1$, and $\left(A A^{*}\right)^{-1}=$ $\left\|A A^{*}\right\|^{-1} \sum_{n=0}^{\infty} R^{n}$. With the method from [27], one obtains

$$
\sum_{k=1}^{\infty}\left|\left(R^{k}\right)_{m, n}\right| \leq \sum_{k=1}^{\infty} \min \left\{r^{k},\left(C_{1}\right)^{k}(2 P)^{k-1} e^{\gamma^{\prime \prime}|m-n|^{\beta}}\right\}
$$

where $\gamma^{\prime \prime} \in\left(0, \gamma^{\prime}\right), C_{1}=1+\frac{C_{A A^{*}}}{\left\|A A^{*}\right\|}$ (with $C_{A A^{*}}$ satisfying $\left|\left(A A^{*}\right)_{m, n}\right| \leq$ $C_{A A^{*}} e^{-\gamma^{\prime}|m-n|^{\beta}}$ for every $\left.m, n \in \mathbb{N}\right)$, and $P=P_{\gamma^{\prime}-\gamma^{\prime \prime}, \beta}=\sum_{j=0}^{\infty} e^{-\left(\gamma^{\prime}-\gamma^{\prime \prime}\right) j^{\beta}}$.

For $\mathrm{k}=0,\left(R^{k}\right)_{m, n}$ equals 1 when $m=n$ and 0 otherwise, so we have $\left|\left(R^{0}\right)_{m, n}\right| \leq$ $e^{-p|m-n|^{\beta}}$ for every $p$.

Fix $|m-n|$ and let $\varepsilon \in(0,1)$. With $K:=C_{1} 2 P(>1)$, let

$$
\begin{aligned}
\Lambda_{|m-n|} & :=\left\{j \in \mathbb{N}: K^{j} e^{-\gamma^{\prime \prime}(1-\varepsilon)|m-n|^{\beta}} \leq r^{j}\right\} \\
& =\left\{j \in \mathbb{N}: j \leq \frac{\gamma^{\prime \prime}(1-\varepsilon)|m-n|^{\beta}}{\ln \left(\frac{K}{r}\right)}\right\} .
\end{aligned}
$$

Denote by $n_{0}$ the highest natural number such that $n_{0} \in \Lambda_{|m-n|}$. Then

$$
\begin{aligned}
\sum_{k=1}^{n_{0}}\left|\left(R^{k}\right)_{m, n}\right| & \leq \frac{1}{2 P} \sum_{k=1}^{n_{0}} K^{k} e^{-\gamma^{\prime \prime}(1-\varepsilon)|m-n|^{\beta}} e^{-\varepsilon \gamma^{\prime \prime}|m-n|^{\beta}} \\
& \leq \frac{1}{2 P} e^{-\varepsilon \gamma^{\prime \prime}|m-n|^{\beta}} \sum_{k=1}^{\infty} r^{k}=\frac{r}{1-r} \frac{1}{2 P} e^{-\varepsilon \gamma^{\prime \prime}|m-n|^{\beta}}
\end{aligned}
$$

For $n>n_{0}$ we have $r^{n}<r^{n_{0}}=e^{n_{0} \ln r}$ and hence,

$$
\sum_{k=n_{0}+1}^{\infty}\left|\left(R^{k}\right)_{m, n}\right| \leq \sum_{k=n_{0}+1}^{\infty} r^{k}=r^{n_{0}+1} \sum_{k=0}^{\infty} r^{k}<\frac{1}{1-r} e^{-\gamma^{\prime \prime}(1-\varepsilon) \frac{\ln (1 / r)}{\ln (K / r)}|m-n|^{\beta}} .
$$

Let

$$
\gamma_{1}=\min \left\{\frac{\ln 1 / r}{\ln K / r} \gamma^{\prime \prime}(1-\varepsilon), \varepsilon \gamma^{\prime \prime}\right\}
$$


Therefore,

$$
\sum_{k=0}^{\infty}\left|\left(R^{k}\right)_{m, n}\right| \leq e^{-\gamma_{1}|m-n|^{\beta}}\left(1+\frac{r}{1-r} \cdot \frac{1}{2 P}+\frac{1}{1-r}\right) .
$$

Now using the representation $A^{-1}=A^{*}\left(A A^{*}\right)^{-1}$ and Lemma 7.1, we can conclude that

$$
\left|\left(A^{-1}\right)_{m, n}\right| \leq C_{A}\left\|A A^{*}\right\|^{-1}\left(1+\frac{r}{1-r} \cdot \frac{1}{2 P}+\frac{1}{1-r}\right) 2 P_{\gamma-\gamma_{1}, \beta} e^{-\gamma_{1}|m-n|^{\beta}},
$$

where $C_{A}$ is a positive constant such that $\left|A_{m, n}\right| \leq C_{A} e^{-\gamma|m-n|^{\beta}}$ for $m, n \in \mathbb{N}$.

\section{References}

1. Balan, R., Casazza, P.G., Heil, C., Landau, Z.: Density, overcompleteness, and localization of frames. Electron. Res. Announc. Am. Math. Soc. 12, 71-86 (2006)

2. Balan, R., Casazza, P.G., Heil, C., Landau, Z.: Density, overcompleteness, and localization of frames. I. Theory. J. Fourier Anal. Appl. 12(2), 105-143 (2006)

3. Balan, R., Casazza, P.G., Heil, C., Landau, Z.: Density, overcompleteness, and localization of frames. II. Gabor systems. J. Fourier Anal. Appl. 12(3), 309-344 (2006)

4. Bari, N.K.: Biorthogonal systems and bases in Hilbert space. Mathematics, Uch. Zap. Mosk. Gos. Univ., 148, vol. IV, pp. 69-107. Moscow Univ Press, Moscow (1951)

5. Balazs, P., Gröchenig, K.: A guide to localized frames and applications to Galerkin-like representations of operators. In: Pesenson, I., Le Gia, Q., Mayeli, A., Mhaskar, H., Zhou, D.X. (eds.) Frames and Other Bases in Abstract and Function Spaces. Applied and Numerical Harmonic Analysis. Birkhäuser, Cham (2017)

6. Bonet, J., Fernández, C., Galbis, A., Ribera, J.M.: Shrinking and boundedly complete Schauder frames in Fréchet spaces. J. Math. Anal. Appl. 410(2), 953-966 (2014)

7. Casazza, P., Christensen, O.: Perturbation of operators and applications to frame theory. J. Fourier Anal. Appl. 3(5), 543-557 (1997)

8. Casazza, P., Christensen, O., Stoeva, D.T.: Frame expansions in separable Banach spaces. J. Math. Anal. Appl. 307, 710-723 (2005)

9. Casazza, P., Han, D., Larson, D.: Frames for Banach spaces. Contemp. Math. 247, 149-182 (1999)

10. Chen, Y., Signahl, M., Toft, J.: Hilbert space embeddings for Gelfand-Shilov and Pilipović spaces. In: Oberguggenberger, M., Toft, J., Vindas, J., Wahlberg, P. (eds.) Generalized Functions and Fourier Analysis. Operator Theory: Advances and Applications, vol. 260. Birkhäuser, Cham (2017)

11. Chen, Y., Signahl, M., Toft, J.: Factorizations and singular value estimates of operators with GelfandShilov and Pilipović kernels. J. Fourier Anal. Appl. 24(3), 666-698 (2018)

12. Christensen, O.: An Introduction to Frames and Riesz Bases. Applied and Numerical Harmonic Analysis, Second Expanded Edition. Birkhäuser, Boston (2016)

13. Christensen, O., Strohmer, T.: The finite section method and problems in frame theory. J. Approx. Theory 133, 221-237 (2005)

14. Cordero, E., Gröchenig, K.: Localization of frames. II. Appl. Comput. Harmon. Anal. 17(1), 29-47 (2004)

15. Duffin, R.J., Schaeffer, A.C.: A class of nonharmonic Fourier series. Trans. Am. Math. Soc. 72, 341-366 (1952)

16. Duran, A.J.: Laguerre expansions of tempered distributions and generalized functions. J. Math. Anal. Appl. 150(1), 166-180 (1990)

17. Duran, A.J.: Laguerre expansions of Gel'fand-Shilov spaces. J. Approx. Theory 74, 280-300 (1993)

18. Feichtinger, H.G.: Gewichtsfunktionen auf lokalkompakten Gruppen. Sitzungsber. Abt. II, Österr. Akad. Wiss. Math.-Naturwiss. K1. 188, 451-471 (1979) 
19. Fernández, C., Galbis, A., Toft, J.: The Bargmann transform and powers of harmonic oscillator on Gelfand-Shilov subspaces. Revista de la Real Academia de Ciencias Exactas, Físicas y Naturales. Serie A. Matemáticas 111(1), 1-13 (2017)

20. Futamura, F.: Banach frames, decay in the context of localization. Sampl. Theory Signal Image Process. 6(2), 151-166 (2007)

21. Fornasier, M., Gröchenig, K.: Intrinsic localization of frames. Construct. Approx. 22(3), 395-415 (2005)

22. Gröchenig, K.: Describing functions: atomic decompositions versus frames. Monatsh. Math. 112(1), $1-42$ (1991)

23. Gröchenig, K.: Localization of frames, Banach frames, and the invertibility of the frame operator. J. Fourier Anal. Appl. 10(2), 105-132 (2004)

24. Gröchenig, K.: Weight functions in time-frequency analysis. In: Rodino L., Schulze B.-W., Wong M. W. (eds.), Pseudo-Differential Operators: Partial differential equations and time-frequency analysis. Fields Inst. Commun. 52, 343-366, American Mathematical Society and The Fields Institute for Research in Mathematical Sciences, Toronto (2007)

25. Jakšić, S., Prangoski, B.: Extension theorem of Whitney type for $\mathcal{S}^{\prime}\left(\mathbb{R}_{+}^{d}\right)$ by the use of the Kernel Theorem. Publ. Inst. Math. 99, 59-65 (2016)

26. Jakšić, S., Pilipović, S., Prangoski, B.: G-type spaces of ultradistributions over $\mathbb{R}_{+}^{d}$ and the Weyl pseudo-differential operators with radial symbols. Revista de la Real Academia de Ciencias Exactas, Físicas y Naturales. Serie A. Matemáticas 111(3), 613-640 (2017)

27. Jaffard, S.: Propriétés des matrices "bien localisées" prés de leur diagonale et quelques applications. Ann. Inst. Henri Poincaré sect. C, Anal. Non linéaire 7(5), 461-476 (1990)

28. Meise, R., Vogt, D.: Introduction to Functional Analysis. Clarendon Press, Oxford (1997)

29. Pilipovic, S.: Generalization of Zemanian spaces of generalized functions which elements have series expansion. SIAM J. Math. Anal. 17, 477-484 (1986)

30. Pilipović, S.: Tempered ultradistributions. Boll. Unione Mat. Ital. 7, 235-251 (1988)

31. Pilipović, S., Stoeva, D.T., Teofanov, N.: Frames for Fréchet spaces. Bull. Cl. Sci. Math. Nat. Sci. Math. 32, 69-84 (2007)

32. Pilipović, S., Stoeva, D.T.: Series expansions in Fréchet spaces and their duals, construction of Fréchet frames. J. Approx. Theory 163, 1729-1747 (2011)

33. Pilipović, S., Stoeva, D.T.: Analysis of conditions for frame functions, examples with the orthogonal functions. Integral Transforms Spec. Funct. 22(4), 311-318 (2011)

34. Pilipović, S., Stoeva, D.T.: Fréchet frames, general definition and expansions. Anal. Appl. 12(2), 195208 (2014)

35. Pilipović, S., Stoeva, D.T.: Frame expansions of test functions, tempered distributions, and ultradistributions. In: Lindahl, K., Lindström, T., Rodino, L.G., Toft, J., Wahlberg, P. (eds.) Analysis, Probability, Applications, and Computation. Trends in Mathematics. Birkhäuser, Basel (2019)

36. Simon, B.: Distributions and their Hermite expansions. J. Math. Phys. 12(1), 140-148 (1971)

37. Stoeva, D.T.: Perturbation of frames in Banach spaces. Asian-Eur. J. Math. 5(1), 1250011 (2012)

38. Guillemot-Teissier, M.: Développements des distributions en séries de fonctions orthogonales. Séries de Legendre et de Laguerre (French). (Development of distributions in series of orthogonal functions. Series of Legendre an Laguerre). Ann. Sc. Norm. Super. Pisa, Sci. Fis. Mat., III. Ser. 25, 519-573 (1971)

Publisher's Note Springer Nature remains neutral with regard to jurisdictional claims in published maps and institutional affiliations. 